\title{
Correlated atomic wires on substrates. II. Application to Hubbard wires
}

\author{
Anas Abdelwahab and Eric Jeckelmann \\ Leibniz Universität Hannover, Institut für Theoretische Physik, Appelstr. 2, 30167 Hannover, Germany \\ Martin Hohenadler \\ Institut für Theoretische Physik und Astrophysik, Universität Würzburg, Am Hubland, 97074 Würzburg, Germany
}

(Dated: August 19, 2021)

\begin{abstract}
In the first part of our theoretical study of correlated atomic wires on substrates, we introduced lattice models for a one-dimensional quantum wire on a three-dimensional substrate and their approximation by quasi-one-dimensional effective ladder models arXiv:1704.07350. In this second part, we apply this approach to the case of a correlated wire with a Hubbard-type electronelectron repulsion deposited on an insulating substrate. The ground-state and spectral properties are investigated numerically using the density-matrix renormalization group method and quantum Monte Carlo simulations. As a function of the model parameters, we observe various phases with quasi-one-dimensional low-energy excitations localized in the wire, namely paramagnetic Mott insulators, Luttinger liquids, and spin- $1 / 2$ Heisenberg chains. The validity of the effective ladder models is assessed by studying the convergence with the number of legs and comparing to the full three-dimensional model. We find that narrow ladder models accurately reproduce the quasi-onedimensional excitations of the full three-dimensional model but predict only qualitatively whether excitations are localized around the wire or delocalized in the three-dimensional substrate.
\end{abstract}

\section{INTRODUCTION}

In the first paper of this series [1, we introduced a three-dimensional (3D) lattice model for a correlated atomic wire deposited on an insulating substrate and showed how to map it onto a two-dimensional (2D) ladder-like lattice that can be approximated by onedimensional (1D) narrow ladder models (NLMs). In this second paper, we apply this approach to a correlated wire represented by the 1D Hubbard model [2] using densitymatrix renormalization group (DMRG) 3 6] and quantum Monte Carlo (QMC) [7, 8] methods. We investigate the occurrence and properties of Luttinger liquids 9-11] and 1D Mott insulators [10, 12] coupled to a substrate.

Atomic wires on surfaces seem to be the ultimate realization of $1 \mathrm{D}$ electron systems 13 15] but the relevance of $1 \mathrm{D}$ physics for these materials is still controversial. In particular, numerous experiments show that some of these materials have gapless excitation spectra with strongly anisotropic charge dynamics. The list of good candidate materials for the realization of (quasi-)1D conductors includes In/Si(111) [15], Au/Ge(100) [16 20, $\mathrm{Bi} / \operatorname{InSb}(100)$ 21, $\mathrm{Pt} / \mathrm{Ge}(100)$ [22, 23, $\mathrm{Pb} / \mathrm{Si}(557)$ [24], and dysprosium silicide nanowires on $\mathrm{Si}(001)$ surfaces 25]. Their properties are sometimes ascribed to Luttinger liquids and sometimes to anisotropic 2D Fermi liquids. One of the main reasons for these controversies is a poor understanding of the influence of the $3 \mathrm{D}$ substrate [13, 15, 26, 28, on 1D conductors. Isolated 1D conductors are known to be Luttinger liquids 9-11, whereas the above experimental realizations raise the question of the stability of Luttinger liquids coupled to an environment [10, 26, 29].

The present theoretical study sheds some light on the quasi-1D physics occurring in correlated atomic wires deposited on semiconducting substrates, in partic- ular on the fate of Luttinger-liquid and Mott-insulating phases when coupled to their environment. In addition, it confirms that few-leg NLMs can describe - at least qualitatively - the quasi-1D low-energy physics of the full $3 \mathrm{D}$ wire-substrate system.

The paper is structured as follows. In Sec. II] we briefly introduce the lattice model for the wire-substrate system and its NLM approximation. The DMRG and QMC methods are outlined in Sec. [II] Results are discussed in Sec. IV and Sec. V contains our conclusions.

\section{MODELS}

\section{A. 3D wire-substrate model}

We consider a wire-substrate model that is a special case of the general model introduced in Sec. II of [1]. It consists of an interacting 1D wire on the surface of a noninteracting insulating $3 \mathrm{D}$ substrate. We use a cubic lattice of size $L_{x} \times L_{y} \times L_{z}$ with the wire aligned in the $x$-direction and open boundary conditions in the $z$-direction. Thus objects on the surface have a coordinate $z=0$. We set all lattice constants and $\hbar$ equal to 1 and therefore do not distinguish between momentum and (dimensionless) wave number.

The system Hamiltonian can be decomposed into three terms describing the substrate degrees of freedom, the wire degrees of freedom, and the coupling between wire and substrate. The 3D substrate is represented by a tight-binding Hamiltonian with a uniform nearestneighbor hopping $t_{\mathrm{s}}>0$ and two orbitals per site with different onsite energies $\pm \epsilon_{\mathrm{s}}\left(\epsilon_{\mathrm{s}}>0\right)$. The resulting single-particle energy spectrum has one valence band $\left(\mathrm{b}=\mathrm{v}, \epsilon_{\mathrm{v}}=-\epsilon_{\mathrm{s}}\right)$ and one conduction band $\left(\mathrm{b}=\mathrm{c}, \epsilon_{\mathrm{c}}=\right.$ 
$\left.+\epsilon_{\mathrm{s}}\right)$ with the dispersion relations

$$
\epsilon_{\mathrm{b}}(\boldsymbol{k})=\epsilon_{\mathrm{b}}-2 t_{\mathrm{s}}\left[\cos \left(k_{x}\right)+\cos \left(k_{y}\right)+\cos \left(k_{z}\right)\right],
$$

where $k_{x}, k_{y} \in[-\pi, \pi]$ and $k_{z} \in[0, \pi]$. The indirect gap between the bottom of the conduction band and the top of the valence band is $\Delta_{\mathrm{s}}=2 \epsilon_{\mathrm{s}}-12 t_{\mathrm{s}}$ and the condition $\Delta_{\mathrm{s}} \geq 0$ requires $\epsilon_{\mathrm{s}}>6 t_{\mathrm{s}}$.

The wire is represented by the 1D Hubbard model [2]. The Hubbard parameter $U \geq 0$ describes the strength of the Coulomb repulsion between two electrons on the same site while a nearest-neighbor hopping term with amplitude $t_{\mathrm{w}}>0$ accounts for the electronic kinetic energy. In addition, an onsite potential $\epsilon_{\mathrm{w}}=-U / 2$ places the Hubbard bands symmetrically around the middle of the substrate band gap. For a noninteracting wire $(U=0)$, we obtain the single-particle dispersion

$$
\epsilon_{\mathrm{w}}\left(k_{x}\right)=-2 t_{\mathrm{w}} \cos \left(k_{x}\right) .
$$

The simplest coupling between the wire and the substrate consists of a hybridization of the electronic orbitals by a hopping term between nearest-neighbor pairs of sites located in the wire and the substrate, respectively. We use the same hybridization strength $t_{\mathrm{ws}}$ for valence and conduction bands.

The total Hamiltonian takes the form

$$
\begin{aligned}
H= & -\frac{U}{2} \sum_{x, \sigma} c_{\mathrm{w} x \sigma}^{\dagger} c_{\mathrm{w} x \sigma}+U \sum_{x} c_{\mathrm{w} x \uparrow}^{\dagger} c_{\mathrm{w} x \uparrow} c_{\mathrm{w} x \downarrow}^{\dagger} c_{\mathrm{w} x \downarrow} \\
& -t_{\mathrm{w}} \sum_{x, \sigma}\left(c_{\mathrm{w} x \sigma}^{\dagger} c_{\mathrm{w}, x+1, \sigma}+\text { H.c. }\right) \\
& +\sum_{b, \boldsymbol{r}, \sigma} \epsilon_{\mathrm{b}} c_{\mathrm{b} \boldsymbol{r} \sigma}^{\dagger} c_{\mathrm{b} \boldsymbol{r} \sigma}-t_{\mathrm{s}} \sum_{\langle\boldsymbol{r} \boldsymbol{q}\rangle} \sum_{\mathrm{b}, \sigma}\left(c_{\mathrm{b} \boldsymbol{r} \sigma}^{\dagger} c_{\mathrm{b} \boldsymbol{q} \sigma}+\text { H.c. }\right) \\
& -t_{\mathrm{ws}} \sum_{b, x, \sigma}\left(c_{\mathrm{b} \boldsymbol{r} \sigma}^{\dagger} c_{\mathrm{w} x \sigma}+\text { H.c. }\right)
\end{aligned}
$$

The sums over $x$ run from 1 to $L_{x}$ with $\boldsymbol{r}=\left(x, y_{0}, 1\right)$ in the last sum ( $y_{0}$ is the $y$-coordinate of the wire), the sum over $\boldsymbol{r}$ runs over all substrate lattice sites, and the sum over $\langle\boldsymbol{r} \boldsymbol{q}\rangle$ is over all pairs of nearest-neighbor sites in the substrate. The operator $c_{\mathrm{b} r \sigma}^{\dagger}$ creates an electron with spin $\sigma$ on the site with coordinates $\boldsymbol{r}=(x, y, z)$ in the substrate orbital $\mathrm{b}=\mathrm{v}, \mathrm{c}$, while $c_{\mathrm{w} x \sigma}^{\dagger}$ creates an electron with spin $\sigma$ on the wire site at $\boldsymbol{r}=\left(x, y_{0}, 0\right)$.

\section{B. 1D narrow ladder models}

As explained in [1, the full 3D wire-substrate system can be mapped exactly onto a ladder-like 2D lattice of size $L_{x} \times N_{\text {imp }}$ with $N_{\text {imp }}=2 L_{y} L_{z}+1$ legs. The explicit form of the full Hamiltonian is

$$
\begin{aligned}
H= & -\frac{U}{2} \sum_{x, \sigma} g_{x 0 \sigma}^{\dagger} g_{x 0 \sigma}+U \sum_{x} g_{x 0 \uparrow}^{\dagger} g_{x 0 \uparrow} g_{x 0 \downarrow}^{\dagger} g_{x 0 \downarrow} \\
& -t_{\mathrm{w}} \sum_{x, \sigma}\left(g_{x 0 \sigma}^{\dagger} g_{x+1,0 \sigma}+\text { H.c. }\right) \\
& -t_{\mathrm{s}} \sum_{n=1}^{N_{\mathrm{imp}}-1} \sum_{x, \sigma}\left(g_{x n \sigma}^{\dagger} g_{x+1, n \sigma}+\text { H.c. }\right) \\
& -\sum_{n=0}^{N_{\mathrm{imp}}-2} t_{n+1}^{\mathrm{rung}} \sum_{x, \sigma}\left(g_{x n \sigma}^{\dagger} g_{x, n+1, \sigma}+\text { H.c. }\right) .
\end{aligned}
$$

Here, $g_{x n \sigma}^{\dagger}$ creates an electron with spin $\sigma$ at position $x$ in the $n$-th leg $\left(n=0, \ldots, N_{\text {imp }}-1\right)$. The first leg $(n=0)$ is identical with the wire, in particular $g_{x 0 \sigma}^{\dagger}=c_{\mathrm{w} x \sigma}^{\dagger}$, while legs $n=1, \ldots, N_{\mathrm{imp}}-1$ correspond to successive shells around the wire and represent the substrate. Hamiltonian (4) consists of the original Hubbard Hamiltonian for the wire, an intra-leg hopping $t_{\mathrm{s}}$ in every substrate leg, and a nearest-neighbor rung hopping $t_{n}^{\text {rung }}$ between substrate legs $n-1$ and $n$. The first two rung hoppings are $t_{1}^{\text {rung }}=\sqrt{2} t_{\mathrm{ws}}$ and $t_{2}^{\text {rung }}=\sqrt{3 t_{\mathrm{s}}^{2}+\epsilon_{\mathrm{s}}^{2}}$. For larger $n, t_{n+1}^{\text {rung }}$ can be computed numerically using the Lanczos algorithm as described in Sec. III of [1. The relation between $c_{\mathrm{b} r \sigma}^{\dagger}$ and $g_{x n \sigma}^{\dagger}$ is also explained there.

The mapping of the 3D wire-substrate model to the 2D ladder-like system is exact but does not yet simplify the problem. Intuitively, however, 1D physics (such as Luttinger liquid behavior) should occur in the wire or in a region of the substrate around the wire. Thus only legs that are close to the wire should be essential for a qualitative description of the 1D low-energy properties. Therefore, we approximate the 3D wire-substrate model by effective NLMs that are obtained by taking only the $N_{\text {leg }} \ll N_{\text {imp }}$ legs closest to the wire into account. The investigation of a noninteracting wire in [1] established that an NLM must include an odd number of legs $N_{\text {leg }} \geq$ 3 to describe a wire on an insulating substrate.

\section{Parameters}

For insulating substrates, we can find model parameters such that the low-energy excitations of the noninteracting wire lie in the substrate band gap. These excitations are then localized on or around the wire and thus form a $1 \mathrm{D}$ electronic subsystem of the full 3D wiresubstrate system. In [1] we showed that this scenario is achieved at half-filling and close to half-filling with a wire hopping $t_{\mathrm{w}}=3$ and the substrate parameters $t_{\mathrm{s}}=1$ and $\epsilon_{\mathrm{s}}=7$. The latter correspond to an indirect gap $\Delta_{\mathrm{s}}=2 \epsilon_{\mathrm{s}}-12 t_{\mathrm{s}}=2$ and a direct gap $\Delta\left(k_{x}\right)=2 \epsilon_{\mathrm{s}}-8 t_{\mathrm{s}}=6$ for a fixed wave number $k_{x}$ in the single-particle excitation spectrum.

The effective substrate band gap $\Delta_{\mathrm{S}}\left(N_{\text {leg }}\right)$ is larger in the NLM but converges to $\Delta_{\mathrm{s}}$ for $N_{\mathrm{leg}} \rightarrow \infty$. For instance, for the three-leg NLM at vanishing wire-substrate 
coupling $t_{\mathrm{ws}}=0$, the substrate is represented by a noninteracting two-leg ladder with single-particle energies

$$
\epsilon\left(k_{x}\right)= \pm t_{2}^{\text {rung }}-2 t_{\mathrm{s}} \cos \left(k_{x}\right) .
$$

Thus $\Delta_{\mathrm{s}}\left(N_{\text {leg }}=3\right)=2 t_{2}^{\text {rung }}-4 t_{\mathrm{s}} \approx 10.4$ is five times larger than the true gap $\Delta_{\mathrm{s}}=2$. We use the above parameters throughout this work and focus on the model properties as a function of the hybridization between wire and substrate $t_{\mathrm{ws}}$ and the strength of the electronelectron interaction $U$.

At half-filling, the $3 \mathrm{D}$ wire-substrate model contains $N_{p}=N_{\text {imp }} L_{x}$ electrons, whereas the NLM contains $N_{p}=N_{\operatorname{leg}} L_{x}$. We focus on half-filled systems and on systems doped away from half-filling by a finite wire doping $y_{\mathrm{w}} \in(-1,1)\left(N_{p}=N_{\mathrm{imp}} L_{x}+y_{\mathrm{w}} L_{x}\right.$ for the $3 \mathrm{D}$ wire-substrate model, or $N_{p}=N_{\operatorname{leg}} L_{x}+y_{\mathrm{w}} L_{x}$ for the NLM). Such a finite wire doping corresponds to a negligible bulk doping of the substrate in the thermodynamic limit $N_{\text {imp }} \gg 1$ but is relevant for quasi-1D conductors embedded in an insulating 3D bulk system, e.g., metallic wires on semiconducting substrates.

\section{METHODS}

\section{A. DMRG}

The DMRG is a powerful method for quasi-1D correlated quantum systems with short-range interactions [36. It can be used to study relatively wide ladder geometries [30] or coupled chains [31, 32]. For such systems, however, it is limited by an exponential increase of CPU time and required memory as a function of the lattice width. Therefore, our DMRG study is necessarily restricted to correlated NLMs with small numbers of legs $N_{\text {leg }}$. Nevertheless, we found that in general the computational effort required for the NLM increases much more slowly with the number of legs than for a similar homogeneous ladder system. Fundamentally, the exponential increase of the computational cost is due to the rapid increase of entanglement with the ladder width. This entanglement is essentially determined by the number of gapless excitation modes in the system (e.g., the number of bands crossing the Fermi energy in a noninteracting system). In a homogeneous ladder model, this number is typically proportional to the ladder width. In the NLM for an insulating substrate, however, this number remains small when $N_{\text {leg }}$ increases because most excitation modes represent gapped transitions between valence and conduction bands. This results in a slower increase of the computational cost with system width.

We used the finite-system DMRG algorithm on lattices with up to $L_{x}=208$ rungs for three-leg ladders and up to $L_{x}=128$ for wider ladders with up to $N_{\text {leg }}=11$ legs. The ladder length $L_{x}$ was always taken to be an even number and open boundary conditions were used in the $x$-direction. Up to $m=2024$ density-matrix eigenstates were kept in our DMRG calculations, yielding discarded weights smaller than $10^{-6}$. We systematically investigated truncation errors by keeping variable numbers of density-matrix eigenstates and extrapolating ground-state energies to the limit of vanishing discarded weights 33. The resulting error estimates are smaller than the symbols in our figures.

Using the DMRG, we calculated the charge gap

$$
\begin{aligned}
E_{\mathrm{c}}=\frac{1}{2} & {\left[E_{0}\left(M_{\uparrow}+1, M_{\downarrow}+1\right)+E_{0}\left(M_{\uparrow}-1, M_{\downarrow}-1\right)\right.} \\
& \left.-2 E_{0}\left(M_{\uparrow}, M_{\downarrow}\right)\right],
\end{aligned}
$$

the spin gap

$$
E_{\mathrm{s}}=E_{0}\left(M_{\uparrow}+1, M_{\downarrow}-1\right)-E_{0}\left(M_{\uparrow}, M_{\downarrow}\right),
$$

and the single-particle gap

$$
\begin{aligned}
E_{\mathrm{p}}=E_{0}\left(M_{\uparrow}+1, M_{\downarrow}\right)+E_{0}\left(M_{\uparrow}-1, M_{\downarrow}\right) & \\
& -2 E_{0}\left(M_{\uparrow}, M_{\downarrow}\right),
\end{aligned}
$$

where $E_{0}\left(M_{\uparrow}, M_{\downarrow}\right)$ denotes the ground-state energy for $M_{\sigma}$ electrons of spin $\sigma$. These gaps are visible in the dynamic charge structure factor, the dynamic spin structure factor, and the single-particle spectral functions calculated with the QMC method discussed below.

Additional information can be inferred from the distribution of charges and spins on the different legs. The total charge on leg $n$ is defined as

$$
C(n)=\left\langle\psi_{\mathrm{GS}}\left|\sum_{x, \sigma} g_{x n \sigma}^{\dagger} g_{x n \sigma}\right| \psi_{\mathrm{GS}}\right\rangle,
$$

while the total spin- $z$ density is defined by

$$
S(n)=\left\langle\psi_{\mathrm{GS}}\left|\sum_{x, \sigma} \sigma g_{x n \sigma}^{\dagger} g_{x n \sigma}\right| \psi_{\mathrm{GS}}\right\rangle .
$$

Here, $\left|\psi_{\mathrm{GS}}\right\rangle$ is the ground state for $M_{\sigma}$ electrons of spin $\sigma$. Additionally, variations $\Delta C(n)$ and $\Delta S(n)$ of these quantities for $M_{\sigma} \pm 1$ indicate whether the lowest charge, spin and single-particle excitations (defined by the above gaps) are mostly localized on the wire or distributed in the substrate.

The actual excess density on the wire is

$$
y_{\text {eff }}=\frac{C(0)}{L_{x}}-1
$$

while the wire doping corresponds to

$$
y_{\mathrm{w}}=\sum_{n=0}^{N_{\mathrm{leg}}-1}\left[\frac{C(n)}{L_{x}}-1\right] .
$$

If all added electrons (or added holes) are localized in the wire then $y_{\mathrm{eff}}=y_{\mathrm{w}}$. However, in general, $\left|y_{\mathrm{eff}}\right|<\left|y_{\mathrm{w}}\right|$ because the doped particles have a finite probability to be in the substrate. We will show below that it is possible 

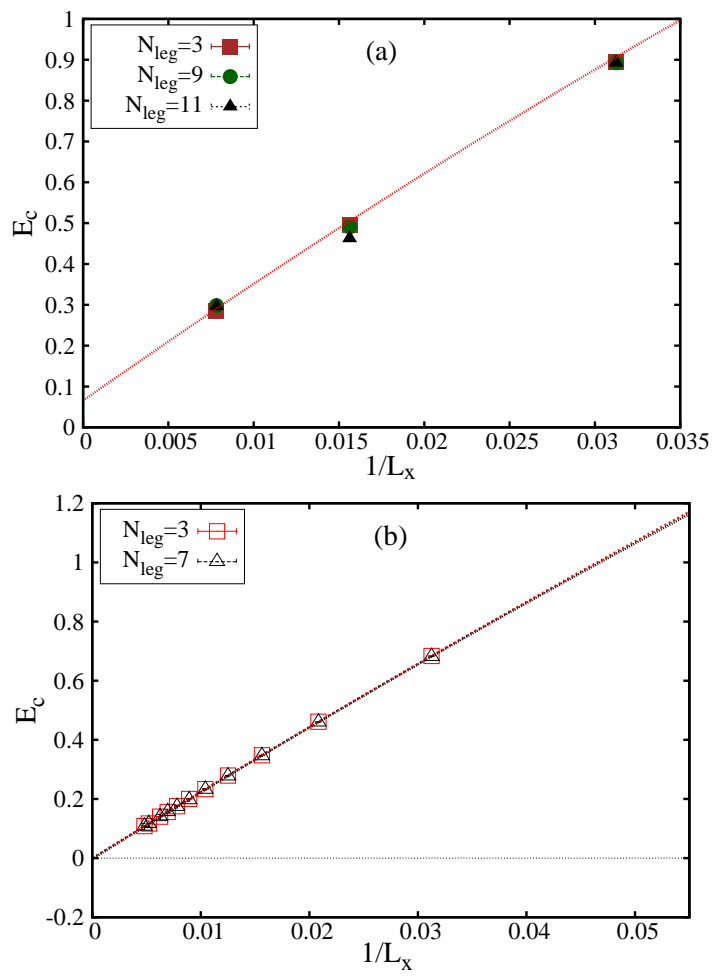

FIG. 1. (Color online) DMRG results for the charge gap [Eq. [6] ] of the NLM (a) at half-filling and (b) away from half-filling $\left(y_{\mathrm{w}}=12.5 \%\right)$ as a function of the inverse ladder length $1 / L_{x}$ for different numbers of legs $N_{\text {leg. }}$. The Hubbard interaction was $U=4$. The line in (a) shows the finite-size gap of the half-filled 1D Hubbard model with open boundary conditions and a hopping $t_{\mathrm{w}}=3$. The lines in (b) correspond to quadratic fits in $1 / L_{x}$.

that they become completely delocalized in the whole substrate so that $\left|y_{\mathrm{w}}\right| \gg\left|y_{\text {eff }}\right| \approx 0$.

To analyze finite-size corrections in the correlated NLM, we calculated these gaps for ladders of various lengths $L_{x}$ and widths $N_{\text {leg. }}$. As a first example, Fig. 11(a) shows the charge gap $E_{\mathrm{c}}$ at half-filling for $U=4$ as a function of $1 / L_{x}$ for different $N_{\text {leg. }}$. It decreases as a function of $1 / L_{x}$ for a fixed $N_{\text {leg. }}$. In 1D Mott insulators, charge and single-particle gaps decrease toward finite values in the limit $L_{x} \rightarrow \infty$. We used a second-order polynomial fit in $1 / L_{x}$ to extrapolate these gaps whenever necessary and possible. In Fig. 1(a) the extrapolated charge gaps are finite and almost equal for all $N_{\text {leg }}$ for the parameters chosen. For other model parameters, we find that the gap can strongly depend on the number of legs and that extrapolations for increasing $N_{\text {leg }}$ at finite system length $L_{x}$ are also unsatisfactory. This complex finitesize scaling is related to the large variation of the effective substrate band gap $\Delta_{\mathrm{s}}\left(N_{\text {leg }}\right)$ with $N_{\text {leg }}$ discussed before for the noninteracting NLM.

As a second example, Fig. 1(b) shows the charge gap away from half-filling $\left(y_{\mathrm{w}}=12.5 \%\right)$ for $U=4$ for two different values of $N_{\text {leg }}$. The charge gap vanishes with
$1 / L_{x}$ for a fixed number of legs. According to conformal field theory, the finite-size gaps of gapless excitations in $1 \mathrm{D}$ electron systems vanish linearly with the inverse of the system length [10,

$$
E_{\alpha}=\frac{\pi v_{\alpha}}{L_{x}}
$$

for $L_{x} \gg 1$, where $\alpha=\mathrm{c}$, s, or $\mathrm{p}$ and $v_{\alpha}$ is the velocity of the corresponding excitation. We can hence calculate the velocities of charge, spin and single-electron excitations from the line slopes in the finite-size-scaling analysis. For the noninteracting wire without a substrate these velocities are equal to the Fermi velocity $v_{\mathrm{F}}=2 t_{\mathrm{w}} \sin \left(k_{\mathrm{F}}\right)$, with $v_{\mathrm{F}}=2 t_{\mathrm{w}}=6$ at half-filling and $v_{\mathrm{F}} \approx 1.96 t_{\mathrm{w}} \approx 5.88$ at $12.5 \%$ doping. We find that the $v_{\alpha}$ do not change significantly with the number of legs for $N_{\text {leg }} \geq 3$, as illustrated in Fig. 1(b) where $v_{\mathrm{c}} / v_{\mathrm{F}} \approx 1.2$. Since our DMRG results for gapless excitation modes are limited to a few values of $N_{\text {leg }}$, we can in principle not rule out more significant finite-size corrections for $N_{\text {leg }}>7$.

Ideally, the finite-size gaps of the NLM should be extrapolated to the thermodynamic limit using a fixed ratio $N_{\text {leg }} / L_{x}$. However, this is not possible with the DMRG because we cannot simulate enough different values of $N_{\text {leg }}$ for fixed $N_{\text {leg }} / L_{x}$. Therefore, the few-leg correlated NLMs accessible to the DMRG are not large enough to accurately investigate the full 2D ladder representation (4) of the wire-substrate system. However, they can yield a useful approximation, as illustrated by our observation that the essential properties (e.g., gapped vs. gapless excitations, or excitations on the wires vs. in the substrate) do not change significantly with $N_{\text {leg }}$ for $N_{\text {leg }} \geq 3$ unless a phase boundary is crossed.

To achieve larger ladder sizes, one could use other DMRG methods and other representations of the NLM that are more appropriate for specific problems. For instance, the two-step DMRG [31, 32] allows one to investigate systems of weakly coupled chains more efficiently. The DMRG can also be used in momentum space [3436, where it yields more accurate results for momentumresolved observables for weak electron-electron interactions. As the NLM is not translationally invariant in the rung direction, however, this approach is not directly applicable. Nevertheless, a clear advantage of the momentum representation of the NLM is that the $y z$-slices of the substrate are decoupled, see Sec. III of 11. It is sufficient to use the momentum representation in the wire direction ( $x$-direction) to achieve this decoupling. Thus one could also envision using a mixed representation $\left(k_{x}, y, z\right)$, i.e., momentum space in the wire direction and real space in the $y$ - and $z$-directions, or $\left(k_{x}, n\right)$, i.e., momentum space in the wire direction and Lanczos basis for the other two directions. DMRG variants that combine momentum and real space have been developed recently to take advantage of such alternative representations [37, 38. The mixed representation $\left(k_{x}, n\right)$ is expected to be the best starting point for field-theoretical approaches [9, 10, 29, 39].

Alternatively, it is possible to consider each $y z$-slice of the substrate (or, equivalently, each rung of the NLM) 
as a single site with a large number of states and apply DMRG methods developed to treat such big sites [4042. This approach may lead to much smaller effective representations of the substrate degrees of freedom because the latter seem to be more weakly entangled than the rungs of homogeneous ladder systems.

\section{B. $\mathrm{QMC}$}

The continuous-time interaction-expansion (CT-INT) QMC method [7] is particularly useful to study both NLMs and the full 3D wire-substrate model. For this purpose, the method is formulated in terms of the fermionic coherent-state path integral with an action $S=S_{0}+S_{1}$. Here, $S_{0}$ is quadratic and has the form

$$
S_{0}=-\sum_{i j \sigma} \iint_{0}^{\beta} d \tau d \tau^{\prime} c_{i \sigma}^{\dagger}(\tau) G_{0, \sigma}^{-1}\left(i-j, \tau-\tau^{\prime}\right) c_{j \sigma}\left(\tau^{\prime}\right),
$$

with the free Green function $G_{0, \sigma}$ describing the hopping between sites $i$ and $j$ of the wire via all possible paths (direct or via the substrate). The Hubbard interaction in the wire is contained in

$S_{1}=U \sum_{i} \int_{0}^{\beta} d \tau\left[c_{i \uparrow}^{\dagger}(\tau) c_{i \uparrow}(\tau)-\frac{1}{2}\right]\left[c_{i \downarrow}^{\dagger}(\tau) c_{i \downarrow}(\tau)-\frac{1}{2}\right]$.

The key idea of the method is a Dyson-expansion of the partition function $Z=\operatorname{tr} e^{-S}$ in powers of $S_{1}$, which can be summed exactly by stochastic sampling of interaction vertices [7]. The algorithmic details have been discussed in detail before 8 . For the present problem, it is essential to understand that interactions are restricted to the wire, whereas substrate sites are noninteracting both in the NLM and the full 3D problem. As in previous work on edge states of topological insulators [43, the numerical effort scales as $n^{3}$, where $n \sim U \beta L_{x}$ is the average expansion order and depends only on the number of correlated sites. It is hence the same as for the 1D Hubbard model. The cubic scaling with $n$ makes CT-INT most useful for weak to intermediate couplings. However, because the noninteracting substrate sites are integrated out, NLMs and full 3D models with the same $L_{x}$ require the same computer time so that detailed comparisons between these different models are possible.

Here, we used a grand-canonical variant of CT-INT with inverse temperature $\beta$. A chemical potential $\mu=0$ corresponds to half-filling, whereas $\mu>0$ gives electrondoped systems with $y_{\mathrm{w}}>0$. The total number of doped electrons was adjusted to $L_{x} / 8=5.25$ (or $y_{\mathrm{w}} \approx 12.5 \%$, as in the DMRG results) by tuning $\mu$. The thermodynamic average of the particle density was calculated exactly for the wire as well as the substrate legs of the three-leg NLM [cf. Eqs. (9) and (12)]. For the 3D wire-substrate model, substrate averages over all sites are not feasible because $G_{0}$ has to be stored for all sites and imaginary times. Therefore, substrate properties were obtained by averaging over the chains at minimal $(y=1, z=1)$ and maximal distance $\left(y=L_{y} / 2, z=L_{z}\right)$ from the wire.

To complement the DMRG results, we calculated spectral properties of the NLM and the 3D wire-substrate system. Specifically, we considered the momentumand energy-resolved single-particle spectral functions, as well as the dynamic charge and spin structure factors. These quantities can be measured in experiments such as angle-resolved photoemission spectroscopy, electronenergy-loss spectroscopy, and inelastic neutron scattering, respectively. In principle, dynamic quantities are also accessible with the DMRG method [44 46] but at a high computational cost and with the additional complication of using pseudo-wave numbers and open boundary conditions (see Refs. 47, 48 for recent works).

We considered the single-particle spectral functions defined in [1, namely the wire spectral function $A_{\mathrm{w}}\left(\omega, k_{x}\right)$, the "substrate" spectral function $A_{\mathrm{s}}\left(\omega, k_{x}\right)$ of the threeleg NLM, and the substrate spectral function $A_{\mathrm{s}}\left(\omega, k_{x}\right)$ corresponding to the average of $A_{\mathrm{s}}\left(\omega, k_{x}, y=y_{0}, z=1\right)$ and $A_{\mathrm{s}}\left(\omega, k_{x}, y=y_{0}+L_{y} / 2, z=L_{z}\right)$.

The dynamic charge $(\alpha=\rho)$ and $\operatorname{spin}(\alpha=\sigma)$ structure factors of the wire are defined as

$$
\begin{aligned}
S_{\alpha}\left(\omega, k_{x}\right)=\frac{1}{Z} \sum_{i j} & \left|\left\langle i\left|\hat{S}_{\alpha}\left(k_{x}\right)\right| j\right\rangle\right|^{2}\left(e^{-\beta E_{i}}+e^{-\beta E_{j}}\right) \\
& \times \delta\left(E_{j}-E_{i}-\omega\right) .
\end{aligned}
$$

with

$$
\begin{aligned}
& \hat{S}_{\rho}\left(k_{x}\right)=\frac{1}{\sqrt{L_{x}}} \sum_{x} e^{i k_{x} x} \sum_{\sigma} c_{\mathrm{w} x \sigma}^{\dagger} c_{\mathrm{w} x \sigma} \\
& \hat{S}_{\sigma}\left(k_{x}\right)=\frac{1}{\sqrt{L_{x}}} \sum_{x} e^{i k_{x} x} \sum_{\sigma} \sigma c_{\mathrm{w} x \sigma}^{\dagger} c_{\mathrm{w} x \sigma} .
\end{aligned}
$$

Here, $|i\rangle$ is an eigenstate with energy $E_{i}$. The above spectral functions were determined from the QMC results for the corresponding single-particle, density-density and spin-spin imaginary-time Green functions with the help of the stochastic maximum entropy method [49].

\section{RESULTS}

\section{A. Insulating wire}

For half-filling and $t_{\mathrm{ws}}=0$, the wire is an exactly halffilled Hubbard chain decoupled from the substrate. The ground state of this 1D model for repulsive interactions is a paramagnetic Mott insulator [2. Therefore, we know that the 3D wire-substrate model and the NLM are Mott insulators if $U>0$ and $t_{\mathrm{ws}}=0$.

Figure 2 shows the charge gap $E_{\mathrm{c}}$ of half-filled threeleg and seven-leg correlated NLMs as a function of the interaction $U$ for $L_{x}=128$ and $t_{\mathrm{ws}}>0$. Finite-size effects are considerable for small charge gaps (i.e., small $U)$ but our finite-size analysis show that $E_{\mathrm{c}}$ is finite in the thermodynamic limit at least for $U \geq 4$, see Fig. 1. 


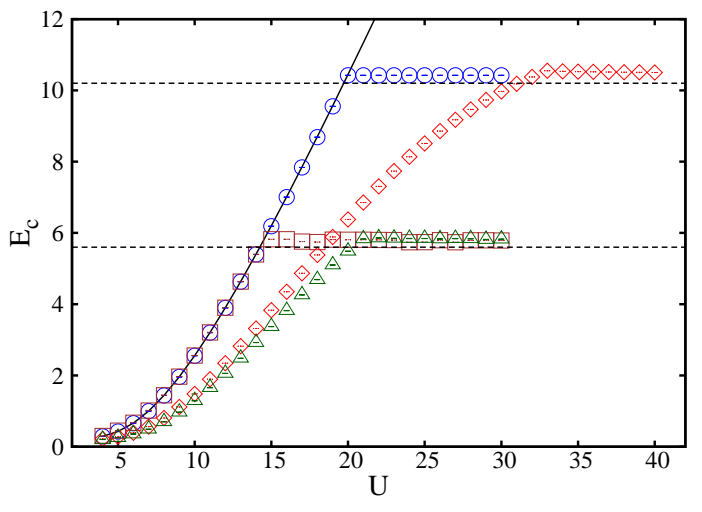

FIG. 2. (Color online) DMRG results for the charge gap [Eq. [6] ] at half-filling as a function of the Hubbard interaction $U$ for a three-leg NLM with $t_{\mathrm{ws}}=0.1$ (circles) and $t_{\mathrm{ws}}=2$ (diamonds), as well as for a seven-leg NLM with $t_{\mathrm{ws}}=0.1$ (squares) and $t_{\mathrm{ws}}=2$ (triangles). The solid line indicates the Mott gap of the 1D Hubbard chain with a hopping $t_{\mathrm{w}}=3$. The horizontal dashed lines indicate the effective substrate band gaps $\Delta_{\mathrm{s}}\left(N_{\text {leg }}=3\right) \approx 10$ and $\Delta_{\mathrm{s}}\left(N_{\text {leg }}=7\right) \approx 5.5$ of the noninteracting NLMs.

For weaker interactions, we could not determine if the charge gap remains finite for $L_{x} \rightarrow \infty$. At stronger interactions, finite-size effects are smaller than the symbol size in Fig. 2. For weak hybridizations $0<t_{\mathrm{ws}} \lesssim 0.5, E_{\mathrm{c}}$ increases with $U$ almost exactly as the Mott gap of a Hubbard chain [2] up to $U_{c} \approx 20$, before saturating abruptly at a value close to the effective substrate band gap for the three-leg NLM $\left[\Delta_{\mathrm{s}}\left(N_{\text {leg }}=3\right) \approx 10\right]$. For stronger wire-substrate hybridization, such as $t_{\mathrm{ws}}=2$ in Fig. 2, the charge gap becomes smaller than the Mott gap of the Hubbard chain but its dependence on $U$ remains qualitatively the same, with saturation occurring at a slightly larger gap value $E_{\mathrm{c}}$ and thus at a larger $U_{c}$. A finite charge gap in the thermodynamic limit and a saturation effect can be observed for hybridizations up to $t_{\mathrm{ws}}=4$. Finally, Fig. 2 shows that the behavior of the gap is qualitatively similar for the three-leg and the seven-leg NLM but that the critical coupling $U_{c}$ decreases with increasing $N_{\text {leg. }}$. The single-particle gap behaves essentially like the charge gap.

Figure 3 shows that the spin gap vanishes linearly with $1 / L_{x}$ in the three-leg NLM, as expected for a 1D MottHubbard insulator or a spin-1/2 Heisenberg chain. This scaling is observed both above and below the charge gap saturation value $U_{c}$. The slopes (i.e., spin velocities) decrease with increasing $t_{\mathrm{ws}}$, suggesting that the effective exchange coupling between spin degrees of freedom becomes weaker. This behavior remains qualitatively similar for larger $N_{\text {leg. }}$.

The charge and spin distributions (9) and 100 of the half-filled ground state are featureless. Figure 4 shows that the variations of these quantities for the lowest excitations provide much more information. The variations of $C(n)$ for one or two added electrons reveal that the

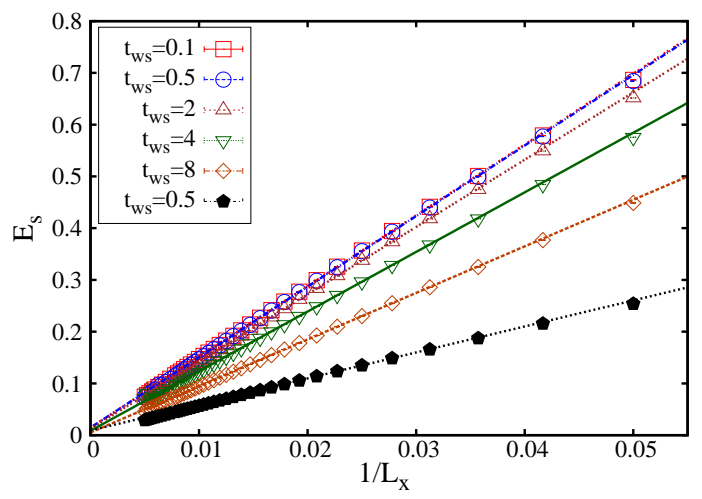

FIG. 3. (Color online) DMRG results for the spin gap [Eq. 77] of the half-filled three-leg NLM as a function of the inverse ladder length for $U=4$ (open symbols) and $U=24$ (filled symbols) and various hybridization strengths $t_{\mathrm{ws}}$.

lowest charge excitations are mostly situated on the wire leg for $U<U_{c}$ but on the noninteracting substrate legs for $U>U_{c}$. The variations of $S(n)$ for a triplet excitation shows that the lowest spin excitations are localized on the wire leg for any $U \geq 4$. In contrast, for a singleparticle excitation (i.e., one added electron), the excess spin goes on the wire leg for $U<U_{c}$ but on the substrate legs for $U>U_{c}$. These uneven distributions are more pronounced for weaker hybridizations $t_{\mathrm{ws}}$. We have verified that they remain qualitatively similar for larger numbers of legs up to $N_{\text {leg }}=7$.

The CT-INT single-particle spectral functions are shown in Fig. 5 for the three-leg NLM and the 3D wiresubstrate model with $t_{\mathrm{ws}}=0.5$ and $U=8$. First, we see that the wire spectral functions $A_{\mathrm{w}}\left(\omega, k_{x}\right)$ in Figs. 5(a) and (b) are very similar despite the significant differences in the substrate spectral functions $A_{\mathrm{s}}\left(\omega, k_{x}\right)$ shown in

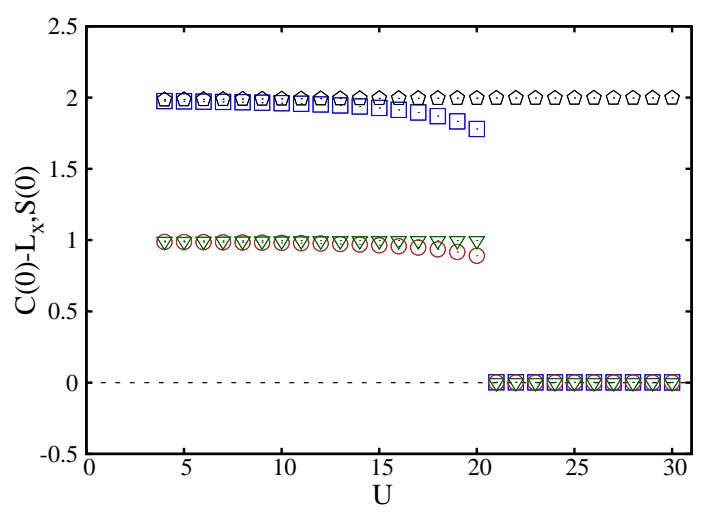

FIG. 4. (Color online) DMRG results for the variation of the total charge $\Delta C(0)=C(0)-L_{x}$ and spin $\Delta S(0)=S(0)$ on the wire leg of the half-filled three-leg NLM as a function of the Hubbard interaction $U$. Here, $t_{\mathrm{ws}}=0.5$. The different symbols correspond to the lowest charge (squares), spin (pentagons), and single-particle (circles and triangles, respectively) excitations. 

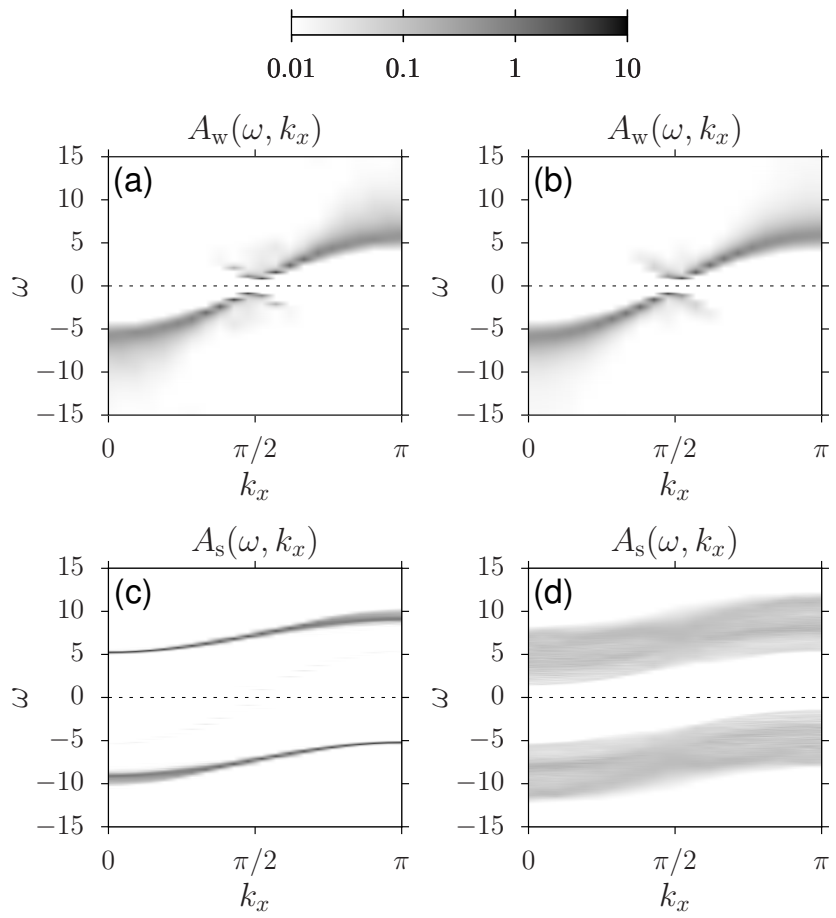

FIG. 5. CT-INT results for the spectral functions $A_{\mathrm{w}}\left(\omega, k_{x}\right)$ $[(\mathrm{a}),(\mathrm{b})]$ and $A_{\mathrm{s}}\left(\omega, k_{x}\right)[(\mathrm{c}),(\mathrm{d})]$ for $U=8, t_{\mathrm{ws}}=0.5, \beta=15$, and $L_{x}=42$. The chemical potential was $\mu=0$, corresponding to half-filling. Panels (a) and (c) show results for the three-leg NLM, panels (b) and (d) for the 3D wire-substrate model $\left(L_{y}=42, L_{z}=10\right)$.

Figs. 5(c) and (d). This confirms that the three-leg NLM can provide a good approximation of the wire properties in the full 3D wire-substrate model. The wire spectral functions closely resemble those of 1D Mott insulators [45, 50 52. A gap is clearly visible in Figs. 55(a) and (b) and its size agrees with the DMRG results of Fig. 2 within the numerical accuracy. The substrate band gap is also clearly seen in the substrate spectral functions $A_{\mathrm{s}}\left(\omega, k_{x}\right)$ in Figs. 5(c) and (d). The gap in the wire spectral function is smaller than the effective substrate gap $\Delta_{\mathrm{S}}\left(N_{\text {leg }}=3\right) \approx 10$ of the three-leg NLM but quite close to the true band gap $\Delta_{\mathrm{s}} \approx 2$ of the $3 \mathrm{D}$ substrate. Finally, Figs. 5(a) and (c) reveal that the spectral weight for the lowest single-particle excitations (i.e., for small $|\omega|)$ of the three-leg NLM is concentrated exclusively in the wire. This confirms that these excitations are localized in the wire in this model for $U<U_{c}$, as suggested by the spin and charge densities of the single-particle excitations in Fig. 4 .

Figure 6 shows the charge and spin structure factors of the wire for the same parameters as in Fig. 5 . These spectra are very similar for the three-leg NLM and the $3 \mathrm{D}$ wire-substrate model, which again supports the validity of the NLM approximation for the 1D physics occurring in the 3D wire-substrate model. The low-energy features seen in $S_{\rho}\left(\omega, k_{x}\right)$ and $S_{\sigma}\left(\omega, k_{x}\right)$ resemble the ones

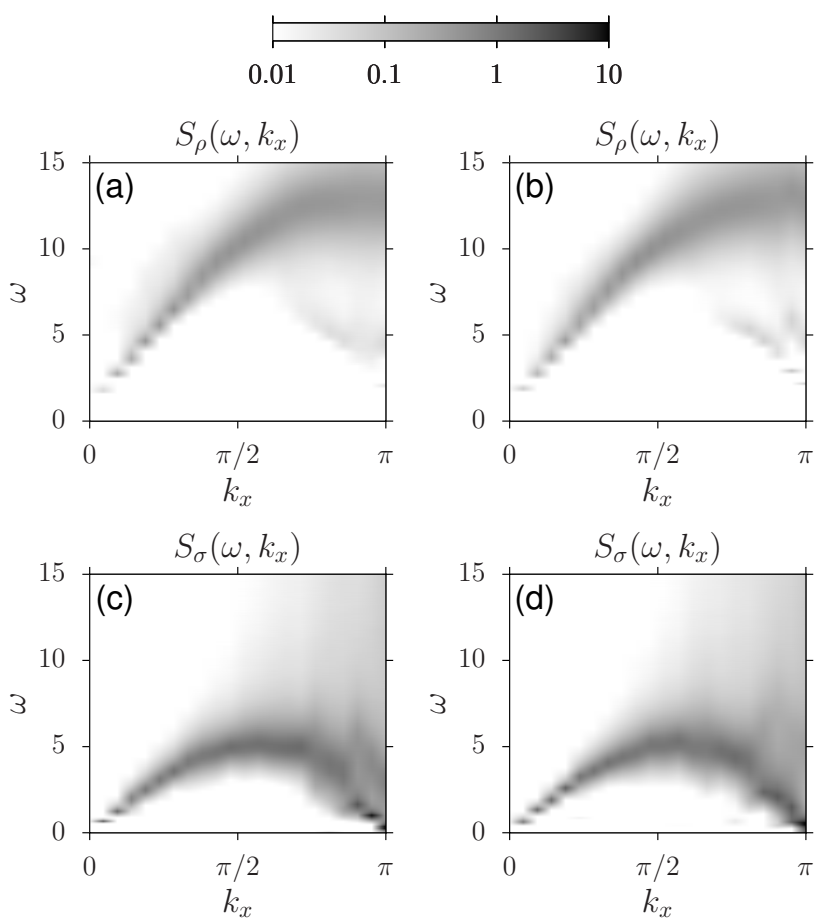

FIG. 6. CT-INT results for the dynamic charge structure factor $S_{\rho}\left(\omega, k_{x}\right)[(\mathrm{a}),(\mathrm{b})]$ and the dynamic spin structure factor $S_{\sigma}\left(\omega, k_{x}\right)[(\mathrm{c}),(\mathrm{d})]$ on the wire for the same parameters as in Fig. 5 Panels (a) and (c) show results for the three-leg NLM, panels (b) and (d) for the 3D wire-substrate model.

found in 1D Mott insulators with gapless spin excitations $45,48,52,53$. The slope of the main feature in the spin structure factor for $k_{x} \rightarrow 0$ agrees with our DMRG results for the velocity of spin excitations.

The spectral properties obtained with the CT-INT method are rather similar for other values of $U$. However, for weaker interactions $U$, we find that the spectral weight of the lowest single-particle excitations is concentrated mostly on the wire not only for the NLM but also for the 3D wire-substrate model. This indicates that the localization of the low-energy single-particle excitations on the 1D wire subsystem is not an artifact of the NLM but a feature of the 3D wire-substrate model in this parameter regime.

Figures 7 and 8 show the spectral functions and dynamic structure factors for $U=12$ (above the estimated critical coupling for the charge gap saturation $U_{c} \approx 9$ of the full $3 \mathrm{D}$ wire-substrate model at $t_{\mathrm{ws}}=0.5$, see below). We again see that the spectral properties of the wire are very similar in the three-leg NLM and the 3D wire-substrate model. In contrast to $U=8$, Figs. 7(b) and $(\mathrm{d})$ reveal that the gap in the single-particle spectral function for the wire $(\Delta \omega \approx 4)$ is comparable to the Mott gap of the 1D Hubbard chain and thus significantly larger than the substrate band gap of the 3D wire-substrate model $\left(\Delta_{\mathrm{S}} \approx 2\right)$. Thus low-energy single-particle excitations now involve the valence and conduction bands and 


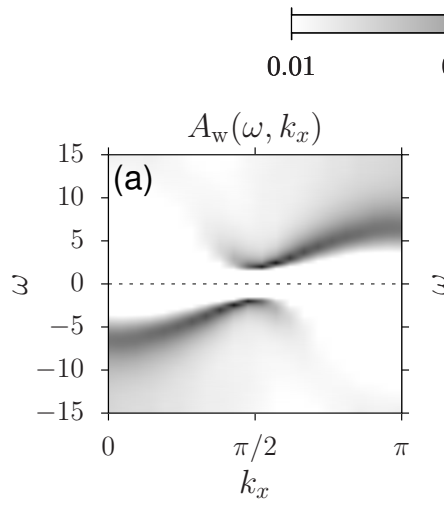

$0.1-1$
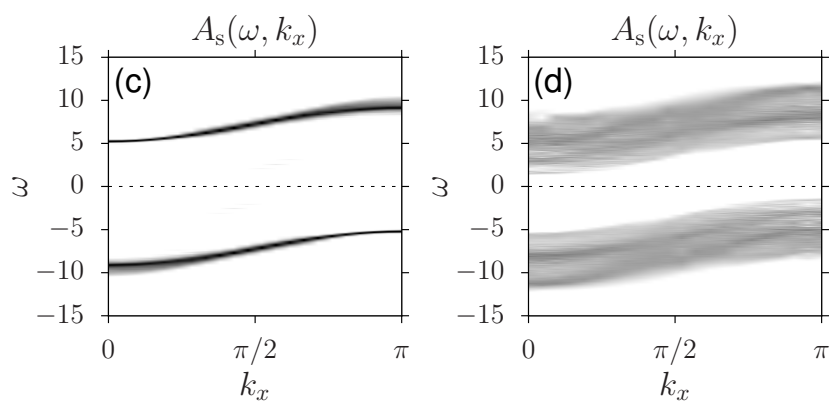

FIG. 7. CT-INT results for the spectral functions $A_{\mathrm{w}}\left(\omega, k_{x}\right)$ $[(\mathrm{a}),(\mathrm{b})]$ and $A_{\mathrm{s}}\left(\omega, k_{x}\right)[(\mathrm{c}),(\mathrm{d})]$ for $U=12, t_{\mathrm{ws}}=0.5, \beta=10$, and $L_{x}=42$. The chemical potential was set to $\mu=0$, corresponding to half-filling. Panels (a) and (c) show results for the three-leg NLM, panels (b) and (d) for the 3D wiresubstrate model $\left(L_{y}=42, L_{z}=10\right)$.

are delocalized in the substrate. This agrees qualitatively with our DMRG results for the three-leg NLM above its critical coupling $U_{c} \approx 20$, i.e., the charge gap saturation in Fig. 2 as well as the spin and charge densities of single-particle excitations in Fig. 4.

Figure 8(b) shows that the dynamic charge structure factor of the wire has no spectral weight at energies between the substrate band gap at $\omega \approx 2$ and the singleparticle gap of the wire at $\omega \approx 4$. This suggests that the system still has charge excitations localized on the wire but only at high energy, i.e., above the Mott gap. Finally, the spin structure factor of the $3 \mathrm{D}$ wire-substrate model in Fig. 8(d) confirms the existence of gapless spin excitations localized on the wire even though the lowest single-particle excitations seem to be in the bandinsulating substrate. This again agrees qualitatively with the DMRG results for the three-leg NLM above its critical coupling $U_{c} \approx 20$, in particular the vanishing of the spin gap illustrated in Fig. 3 and the density distribution for spin excitations shown in Fig. 4.

We conclude that the three-leg NLM describes a quasi1D Mott insulator with gapless spin excitations for weak Hubbard interaction $U<U_{c}$, at least for $t_{\mathrm{ws}} \lesssim 4$ and $U \gtrsim 4$. Increasing $t_{\mathrm{ws}}$ reduces the charge gap, and thus the effective repulsion between charges, but also reduces the spin velocity and thus the effective spin exchange

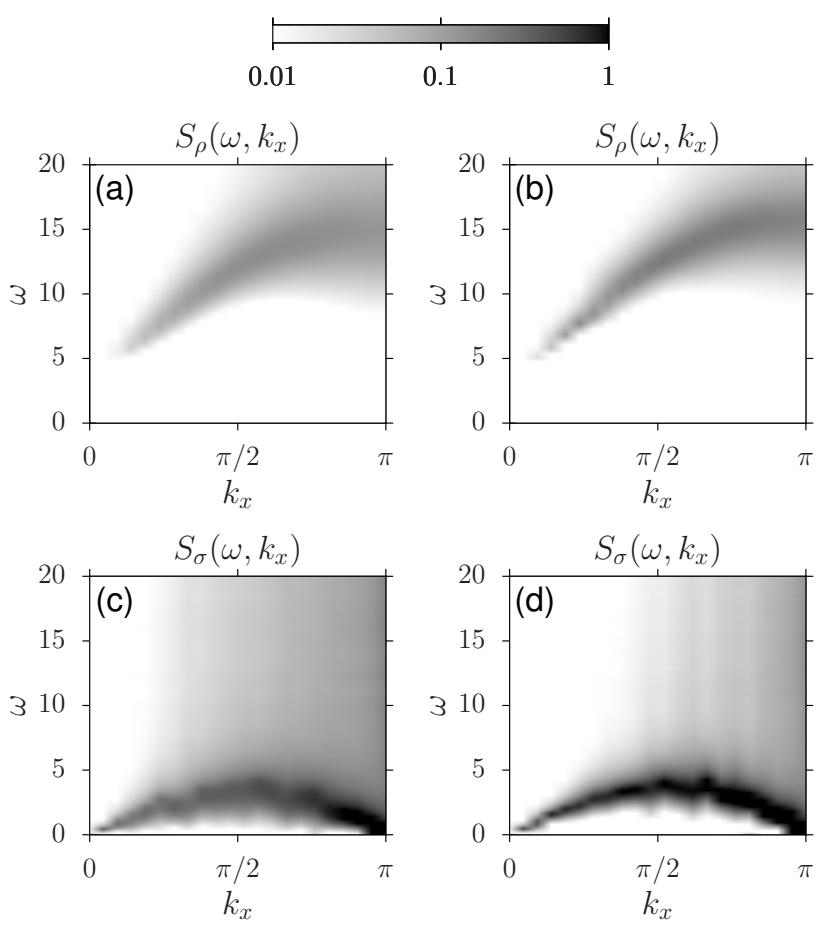

FIG. 8. CT-INT results for the dynamic charge structure factor $S_{\rho}\left(\omega, k_{x}\right)[(\mathrm{a}),(\mathrm{b})]$ and the dynamic spin structure factor $S_{\sigma}\left(\omega, k_{x}\right)[(\mathrm{c}),(\mathrm{d})]$ on the wire for the same parameters as in Fig. 7 Panels (a) and (c) show results for the three-leg NLM, panels (b) and (d) for the 3D wire-substrate model.

coupling compared to an isolated Hubbard chain. Thus, we cannot explain the properties of this phase by a $1 \mathrm{D}$ model with an effective onsite Hubbard interaction only because, in the latter, the effective exchange coupling decreases as the charge gap increases [2].

For strong Hubbard interactions $U>U_{c}$ we find a transition to a band insulator. The lowest charge and single-particle excitations are then transitions between the valence and conduction bands of the NLM representations, as shown by their energy in Fig. 2 and their density variations in Fig. 4. In addition, the spins of the electrons localized on the wire leg represent magnetic impurities embedded in the band insulator. They form an effective Heisenberg chain with the gapless excitations seen in Fig. 3. Unfortunately, the spectral properties in this regime are not accessible by the CT-INT method because of the large expansion orders for $U>U_{c} \approx 20$.

The mechanism of the transition can be understood for weak hybridization $t_{\mathrm{ws}}$ starting from the noninteracting limit discussed in [1. The effective electron-electron interaction in the wire opens a Mott gap in the middle of the wire band as seen in Figs. 5(a) and (b). This gap grows with increasing $U$ until it reaches the effective gap between the substrate bands. For even larger $U$, the band gap is smaller than the Mott gap and the nature of the elementary excitations changes from holons and spinons in a quasi-1D Mott insulator to electrons and holes in a 
band insulator. Indeed, for weak hybridization $t_{\mathrm{ws}}$, the effective Mott gap is almost equal to the gap of the 1D Hubbard chain and the transition occurs exactly when this gap equals the effective band gap of the noninteracting NLM, $\Delta_{\mathrm{s}}\left(N_{\text {leg }}=3\right) \approx 10$ or $\Delta_{\mathrm{s}}\left(N_{\text {leg }}=7\right) \approx 5.5$, see Fig. 2. It is remarkable that this scenario remains qualitatively unchanged up to at least $t_{\mathrm{ws}}=4$.

As discussed above, the effective substrate gap $\Delta_{\mathrm{s}}\left(N_{\text {leg }}\right)$ is considerably reduced upon increasing $N_{\text {leg }}$ until it reaches the value of the true substrate band gap. Accordingly, $U_{c}$ decreases for higher numbers of legs, as seen in Fig. 2. Although we cannot simulate large enough correlated NLMs to observe the convergence of $U_{c}$ with $N_{\text {leg }}$, we expect that it remains finite in the full $3 \mathrm{D}$ wire-substrate system with a finite band gap. Using the criterion discussed above for weak hybridization $t_{\mathrm{ws}}$ (i.e., the $1 \mathrm{D}$ Hubbard gap equals the substrate band gap $\Delta_{\mathrm{s}}=2$ ), we can estimate from Fig. 2 that $U_{c} \approx 9$ in the full $3 \mathrm{D}$ wire-substrate system for small $t_{\mathrm{ws}}$ and that $U_{c}$ becomes larger for stronger hybridization. This interpretation agrees perfectly with the spectral properties computed with the CT-INT method for the 3D wire-substrate model. In particular, the single-particle spectral functions in Figs. 7(b) and (d) demonstrate that this system is a band insulator for $U=12$ while the corresponding structure factors in Figs. 8(b) and (d) confirm that a 1D subsystem with high-energy charge excitations $(\Delta \omega \gtrsim 4)$ but gapless spin excitations (like a spin-1/2 Heisenberg chain) is embedded in that band insulator. Therefore, we think that the transition from the quasi1D Mott insulator to a band insulator is not an artifact of the three-leg NLM but a feature of the correlated 3D wire-substrate model that is qualitatively reproduced by the approximate NLM.

It is known 22 that the half-filled 1D Hubbard model undergoes a phase transition from a metallic Fermi gas at $U=0$ to a Mott insulator for $U>0$. Although we cannot distinguish these phases numerically for very weak $U$, we expect that a similar transition occurs in the NLM and hence in the $3 \mathrm{D}$ wire-substrate model. However, this should be confirmed by methods that are better suited for the weakly interacting regime, such as field-theoretical approaches [9-11, 29, 39] for the three-leg NLM.

\section{B. Metallic wire}

We now turn to the discussion of doped systems. For DMRG calculations we focused on the case of $L_{x} / 8$ added electrons, corresponding to a wire doping of $y_{\mathrm{w}}=12.5 \%$. Removing electrons gives similar results due to electronhole symmetry. Similarly, in the QMC simulations, the chemical potential was tuned to obtain $y_{\mathrm{w}} \approx 12.5 \%$. Due to the different size of the substrate band gap, different chemical potentials were required for the three-leg NLM and the $3 \mathrm{D}$ wire-substrate model. The corresponding values of $\mu$ are given in the figure captions.

For $t_{\mathrm{ws}}=0$ (and $U<\Delta_{\mathrm{s}}$ ), the wire corresponds to a

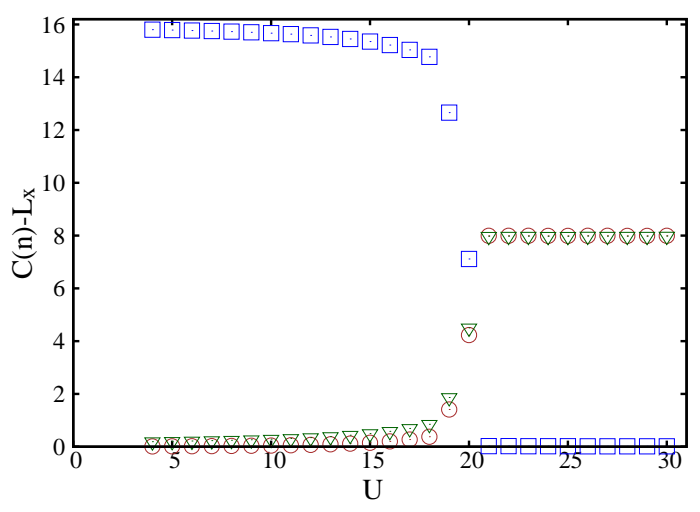

FIG. 9. (Color online) DMRG results for the difference between the total charge away from half-filling $C(n)$ and at half-filling $C(n)=L_{x}$ in the wire leg (squares) as well as in the first (circles) and second (triangles) substrate legs as a function of the interaction $U$. The data are for a three-leg NLM with length $L_{x}=128, t_{\mathrm{ws}}=0.5$ and a wire doping $y_{\mathrm{w}}=12.5 \%$.

doped Hubbard chain. The ground state in this case is a $1 \mathrm{D}$ conductor [2] with the low-energy properties of a Luttinger liquid [10]. We expect these systems to be quasi$1 \mathrm{D}$ conductors also for $t_{\mathrm{ws}} \neq 0$ and to yield information that could be relevant for understanding the numerous metallic atomic wires studied experimentally [15, 25].

Figure 9 shows the variations of the charge distributions $C(n)$ relative to half-filling as a function of the interaction $U$. For weak $U$, most of the added charges go on the wire leg $\left(y_{\mathrm{eff}} \approx y_{\mathrm{w}}\right)$ while for strong $U$ they go on the substrate legs $\left(y_{\text {eff }} \ll y_{\mathrm{w}}\right)$. The crossover-which seems to be continuous but abrupt-occurs close to the critical $U_{c} \approx 20$ found at half-filling and the charge distribution is consistent with the transition from a Mott to a band insulator observed at half-filling. The added electrons occupy states corresponding to the lowest excited states, i.e., in the upper Hubbard band localized on the wire for $U \lesssim U_{c}$ but in the conduction band localized on the substrate legs for $U \gtrsim U_{c}$.

Away from half-filling, the charge, spin and singleparticle gaps vanish in the thermodynamic limit for any $U \geq 0$. However, the doped Mott and band insulating phases exhibit significantly different finite-size effects, as illustrated in Fig. 10 for $U=16$ and $U=24$. For $U \lesssim U_{c}$, the finite-size gaps vanish linearly with $1 / L_{x}$. The velocities defined by Eq. 130 (i.e., the fitted slopes in Fig. 10p are larger for charge excitations $\left(v_{\mathrm{c}}\right)$ than for spin excitations $\left(v_{\mathrm{s}}\right)$ and about the average of $v_{\mathrm{c}}$ and $v_{\mathrm{s}}$ for singleparticle excitations $\left(v_{\mathrm{p}}\right)$. For $U \gtrsim U_{c}$, the charge, spin and single-particle gaps are equal (within the DMRG errors) and much smaller than for weak interactions. The relative DMRG errors for these gaps are too large to accurately determine their scaling with $1 / L_{x}$.

We systematically investigated the velocities of elementary excitations in the weak-coupling phase $\left(U \lesssim U_{c}\right)$ of the three-leg NLM. As expected, the results approach 


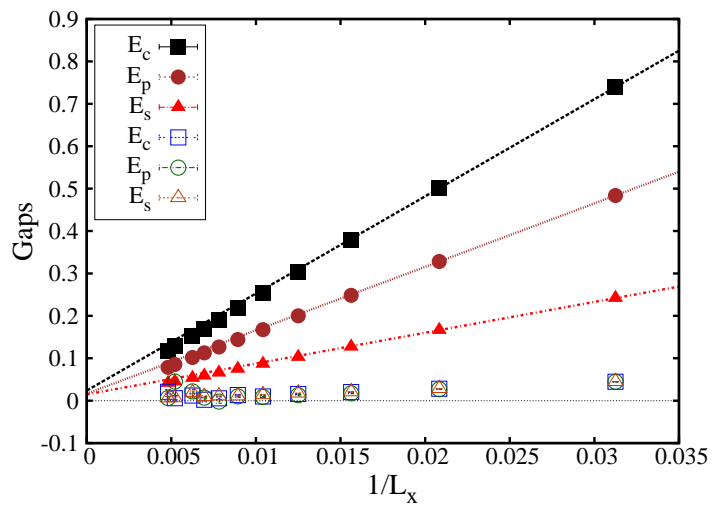

FIG. 10. (Color online) DMRG results for the charge $\left(E_{\mathrm{c}}\right)$, spin $\left(E_{\mathrm{s}}\right)$, and single-particle $\left(E_{\mathrm{p}}\right)$ gaps of the three-leg NLM away from half-filling $\left(y_{\mathrm{w}}=12.5 \%\right)$. Here, $t_{\mathrm{ws}}=0.5$ and $U=$ 16 (filled symbols) and $U=24$ (open symbols), respectively. Slanting lines correspond to linear fits.

those for the 1D Hubbard model when $t_{\mathrm{ws}}$ becomes very small. Although fitting the finite-size DMRG gaps introduces uncertainties, we can recognize two trends in Fig. 11. First, the velocities decrease with increasing wire-substrate hybridization $t_{\mathrm{ws}}$. Second, as observed in the 1D Hubbard model, spin velocities are significantly reduced upon increasing $U$ whereas charge velocities are only weakly affected. Similar to half-filling, we verified that these velocities do not differ significantly in wider NLMs with up to $N_{\text {leg }}=7$. The different charge and spin velocities are a signature of dynamic spin-charge separation typical of the Luttinger liquid state obtained by doping a $1 \mathrm{D}$ Mott insulator. However, for $t_{\mathrm{ws}}>0.5$, the dependence of the velocities on $U$ is different from a doped 1D Hubbard model and thus cannot be captured by an effective onsite interaction only.

Additionally, we investigated the location of the lowest charge and spin excitations in the doped three-leg NLM. Figure 12 shows the variations of charge and spin distributions between excited states and the ground state, similar to Fig. 4 for half-filling. In the weak-coupling regime $U \lesssim U_{c}$, charge, spin and single-particle excitations are almost entirely localized on the wire leg for moderate wire-substrate hybridization strengths (e.g., $t_{\mathrm{ws}}=0.5$ ), similar to the half-filled case. (For larger $t_{\mathrm{ws}}$, charge and spin excitations can be partially localized on both wire and substrate legs and the dependence on $U \lesssim U_{c}$ is more complex.) In contrast, in the strong-coupling regime $U \gtrsim U_{c}$, low-energy excitations are predominantly localized on the substrate legs. Again, we have checked that these uneven distributions persist in wider NLMs with up to $N_{\text {leg }}=7$ legs.

Finally, it should be noted that in the strong-coupling phase the wire is still present as a quasi-1D correlated impurity embedded in the substrate, similar to half-filling. This is clearly visible from the behavior of charge and spin density correlations along the wire. The charge cor-
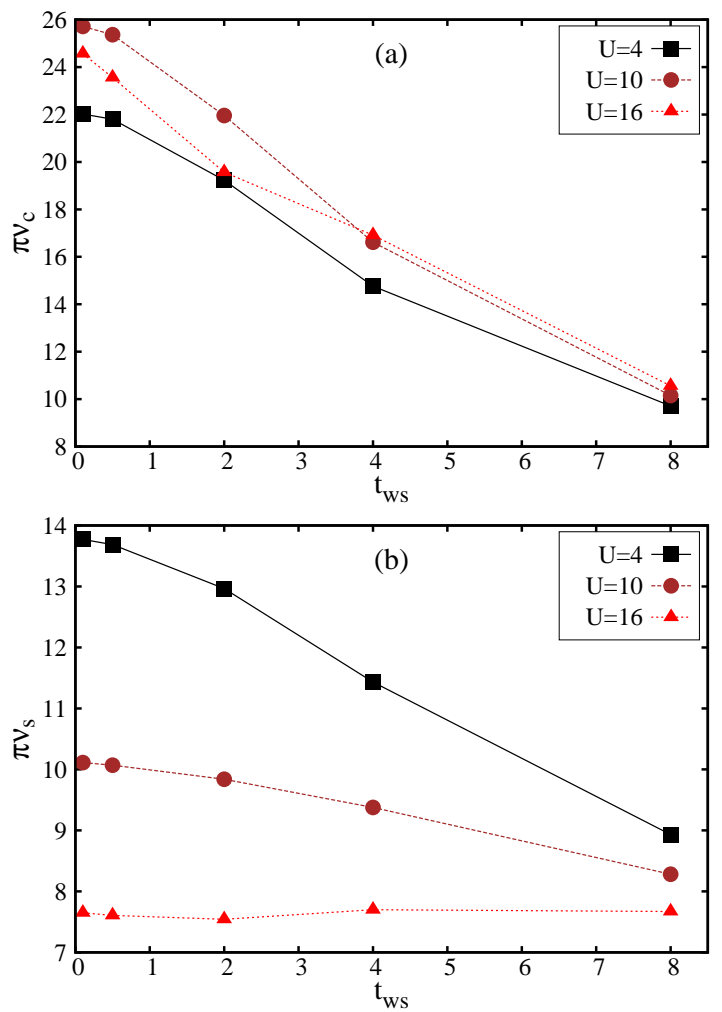

FIG. 11. (Color online) DMRG results for (a) charge and (b) spin velocities of the doped three-leg NLM $\left(y_{\mathrm{w}}=12.5 \%\right)$ as a function of the wire-substrate hybridization strength $t_{\mathrm{ws}}$ for three values of the Hubbard interaction $U$. The results were obtained from the finite-size scaling of the corresponding excitations gaps [cf. Eq. [13]].

relation function for the wire is defined by

$$
\begin{aligned}
F_{\mathrm{c}}\left(x-x^{\prime}\right)= & \left\langle\sum_{\sigma} n_{w x \sigma} \sum_{\sigma^{\prime}} n_{w x^{\prime} \sigma^{\prime}}\right\rangle \\
& -\left\langle\sum_{\sigma} n_{w x \sigma}\right\rangle\left\langle\sum_{\sigma^{\prime}} n_{w x^{\prime} \sigma^{\prime}}\right\rangle
\end{aligned}
$$

while the spin correlation function is

$$
F_{\mathrm{s}}\left(x-x^{\prime}\right)=\left\langle\sum_{\sigma} \sigma n_{w x \sigma} \sum_{\sigma^{\prime}} \sigma^{\prime} n_{w x^{\prime} \sigma^{\prime}}\right\rangle
$$

with $n_{w x \sigma}=c_{\mathrm{w} x \sigma}^{\dagger} c_{\mathrm{w} x \sigma}=g_{x 0 \sigma}^{\dagger} g_{x 0 \sigma}$. Here, expectation values are with respect to the ground state $\left|\psi_{\mathrm{GS}}\right\rangle$. These correlation functions are shown in Fig. 13 for $U=24$ in the strong-coupling phase $\left(t_{\mathrm{ws}}=0.5, U_{c} \approx 20\right)$ and in the weak-coupling phase $\left(t_{\mathrm{ws}}=2.0, U_{c} \approx 32\right)$. In the former case, doped particles populate the substrate, as discussed above, whereas the wire sites are still occupied by one electron on average - as in the half-filled Hubbard model - despite the doping of the three-leg NLM. Accordingly, Fig. 13(a) shows that charge density correlations in the wire decay exponentially for short distances 


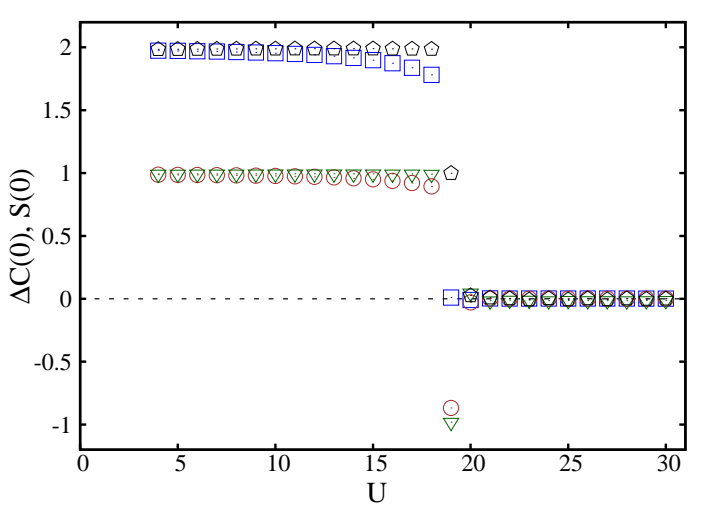

FIG. 12. (Color online) DMRG results for the variations of charge $[\Delta C(0)]$ and spin $[\Delta S(0)=S(0)]$ on the wire leg as a function of $U$ for the doped three-leg NLM $\left(y_{\mathrm{w}}=12.5 \%\right)$. The different symbols correspond to the lowest charge (squares), spin (pentagons), and single-particle (circles and triangles, respectively) excitations.

$x$ in quantitative agreement with the half-filled Hubbard model with the same $U$. [The saturation of $F_{\mathrm{c}}(x)$ at long distances is due to DMRG errors and additional interference from the power-law correlations in the substrate legs.] Similarly, Fig. 13(b) shows that spin correlations in the wire decay with a power-law with an exponent close to -1 , in quantitative agreement with the half-filled Hubbard model. In contrast, in the weak-coupling phase, doped particles populate the wire, resulting in an average density different from one electron per wire site. Correspondingly, the NLM exhibits a power-law decay of charge and spin density correlations, in qualitative agreement with the behavior of these correlation functions in a Hubbard chain with a similar doping $\approx 12.5 \%$ (also shown in Fig. 13).

Additional evidence for two distinct phases comes from the spectral properties calculated with the CTINT method. Figure 14 shows the single-particle spectral functions of the three-leg NLM and the 3D wiresubstrate model for a finite wire doping. The model parameters are the same as in Fig. 5 for half-filling. A Hubbard parameter $U=8$ puts the system in the Luttinger liquid region according to the DMRG results. The wire spectral functions are almost identical for the threeleg NLM [Fig. 14(a)] and the 3D wire-substrate model [Fig. 14(b)]. They are qualitatively similar to those of the doped 1D Hubbard model [44, 46, 54] and compatible with the field-theoretical predictions for Luttinger liquids [55, 56]. In particular, they clearly show the presence of gapless single-particle excitations. In contrast, Fig. 14(c) does not reveal any low-energy excitations in the substrate spectral function of the three-leg NLM. In Fig. 14(d) the Fermi energy (i.e, $\omega=\mu$ ) still lies in the substrate band gap but very close to the bottom of the conduction band and the little spectral weight at $\omega=\mu$ is due to the finite temperature $\beta^{-1}$ used in the QMC simulations. Therefore, the CT-INT single-particle spec-
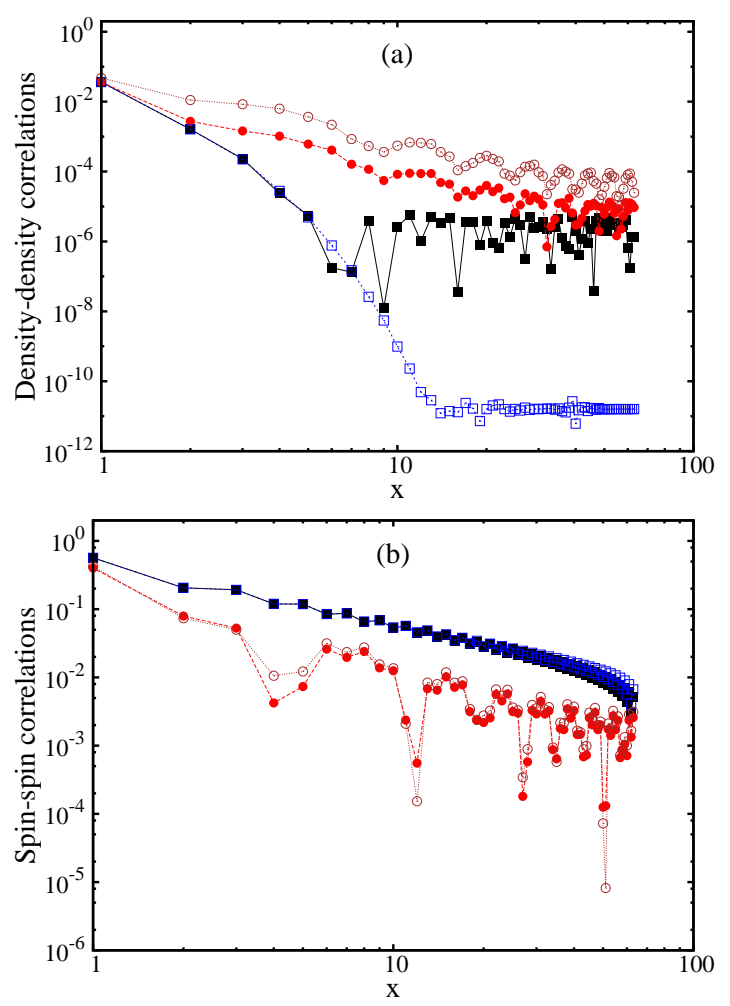

FIG. 13. (Color online) DMRG results for the absolute values of the (a) charge and (b) spin correlation functions on the wire leg for the doped three-leg NLM $\left(y_{\mathrm{w}}=12.5 \%\right)$. Here, $t_{\mathrm{ws}}=0.5$ (filled squares) and $t_{\mathrm{ws}}=2$ (filled circles), respectively. Also shown are results for the 1D Hubbard model with $t_{\mathrm{w}}=3$ at half-filling (open squares) and at $12.5 \%$ doping (open circles). All results are for $U=24$.

tral functions corroborate the existence of gapless lowenergy excitations localized in the wire predicted by the DMRG results. Moreover, they confirm that the threeleg NLM can describe such excitations as well as the 3D wire-substrate model.

The corresponding dynamic charge and spin structure factors of the wire are shown in Fig. 15. Again we see that the spectra are similar for the three-leg NLM and the $3 \mathrm{D}$ wire-substrate model. The structure factors resemble those of the 1D doped Hubbard model 54 and exhibit the features that are expected for electronic Luttinger liquids. Spin and charge excitations are gapless with linear dispersions $\omega=v_{\mathrm{c}, \mathrm{s}} k_{x}$ at low energy. The charge and spin velocities deduced from the CT-INT spectra are compatible with those obtained with the DMRG (see Fig. 11).

The DMRG results for the three-leg NLM revealed a crossover between two conducting phases upon increasing $U$ at fixed doping $y_{\mathrm{w}}$. This crossover has not been investigated directly with the CT-INT method because the critical coupling $U_{c} \approx 20$ is too large. On the other hand, the critical coupling $U_{c} \approx 9$ in the $3 \mathrm{D}$ wire-substrate model is small enough to carry out CT-INT simulations. In that case, however, the chemical potential $\mu$ must be 


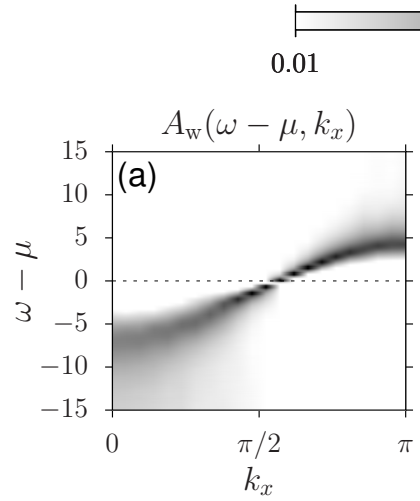

0.1
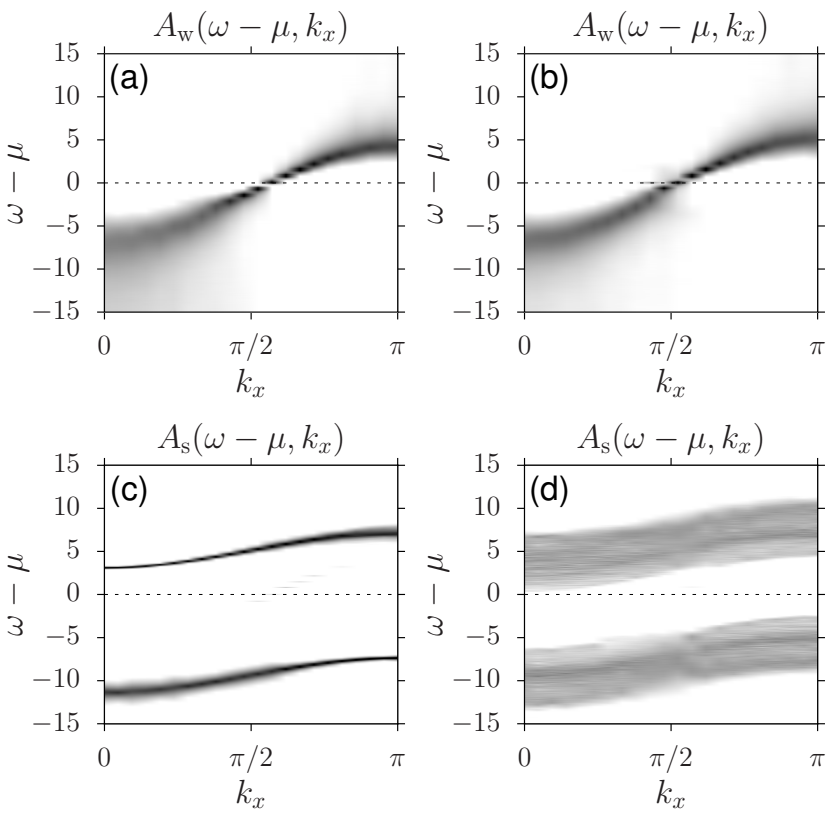

FIG. 14. CT-INT results for the spectral functions $A_{\mathrm{w}}\left(\omega, k_{x}\right)$ $[(\mathrm{a}),(\mathrm{b})]$ and $A_{\mathrm{s}}\left(\omega, k_{x}\right)[(\mathrm{c}),(\mathrm{d})]$ for $U=8, t_{\mathrm{ws}}=0.5, \beta=15$, and $L_{x}=42$. Panels (a) and (c) show results for the threeleg NLM, panels (b) and (d) for the 3D wire-substrate model $\left(L_{y}=42, L_{z}=10\right)$. The chemical potential was $\mu=2.1375$ for the NLM and $\mu=0.99$ for the 3D model, corresponding to a doping of $y_{\mathrm{w}} \approx 12.5 \%$.

just above the lower edge of the conduction band (or, equivalently, just below the upper edge of the valence band) to achieve a finite wire doping $y_{\mathrm{w}}$ but a vanishing dopant density in the wire $y_{\text {eff }}$, i.e., $|\mu| \gtrsim \Delta_{\mathrm{s}} / 2$ for $U=0$. Finding the correct value of $\mu$ for $U>0$ turned out to be a rather delicate problem.

As an example, Fig. 16 shows the single-particle spectral functions of the three-leg NLM and the 3D wiresubstrate model away from half-filling for $U=12$. This interaction is below the critical value $U_{c} \approx 20$ of the three-leg NLM determined with DMRG but above the estimated critical value $U_{c} \approx 9$ for the $3 \mathrm{D}$ wire-substrate model. (The other parameters are equal to those used in Fig. 7 for half-filling.) Accordingly, we see that the spectral functions of the three-leg NLM are qualitatively similar to those for $U=8$ in Fig. 14. For the 3D wiresubstrate model, however, Fig. 16.(b) shows that the wire spectral function resembles that for half-filling in Fig. 7. The Fermi energy still lies within the Hubbard gap, close to the bottom of the upper Hubbard band. In addition, Fig. 16(d) confirms that the Fermi energy lies at the edge of the conduction band. This corresponds to a doped band insulator with gapless single-particle excitations delocalized in the full substrate. The density of charge carriers is $y_{\mathrm{w}} / N_{\text {leg }}$ in the NLM and thus vanish-

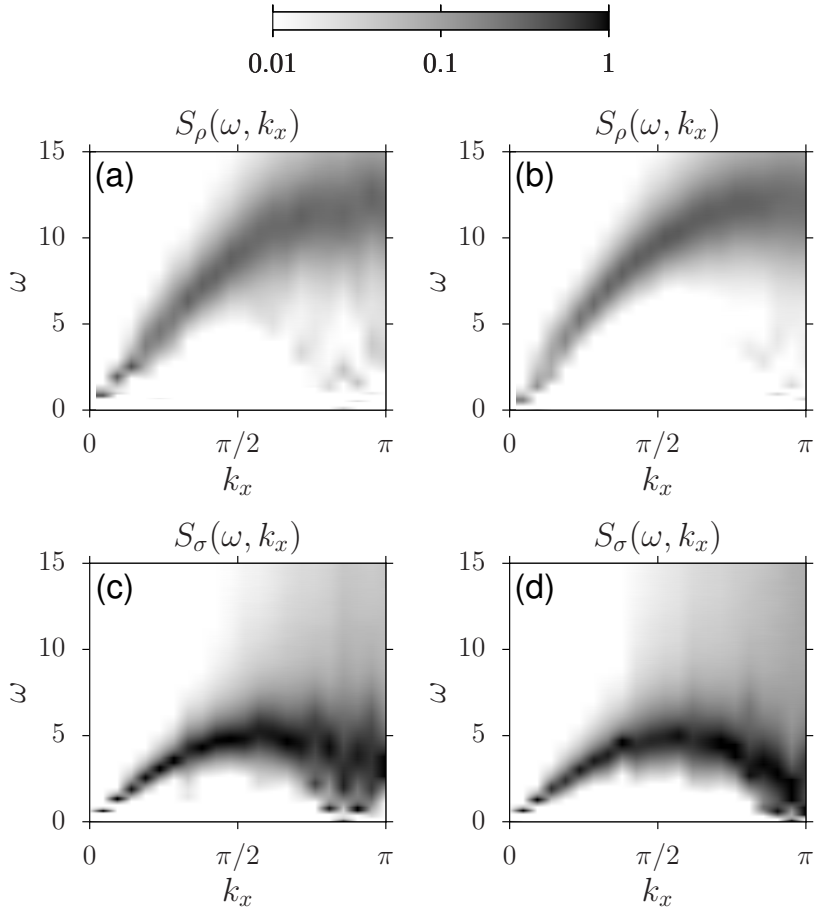

FIG. 15. CT-INT results for the dynamic charge structure factor $S_{\rho}\left(\omega, k_{x}\right)[(\mathrm{a}),(\mathrm{b})]$ and the dynamic spin structure factor $S_{\sigma}\left(\omega, k_{x}\right)[(\mathrm{c}),(\mathrm{d})]$ on the wire for the same parameters as in Fig. 14. Panels (a) and (c) show results for the three-leg NLM, panels (b) and (d) for the 3D wire-substrate model.

ingly small in the $3 \mathrm{D}$ wire-substrate model.

Figure 17 shows the dynamic charge and spin structure factors of the wire for the same parameters as in Fig. 16. Figures 17(a) and (b) confirm that charge excitations in the wire are gapless for the three-leg NLM but have a gap equal to the Mott gap of the half-filled 3D wire-substrate model, cf. Fig. 8(b). The spin excitations are gapless and the spin structure factors are very similar in both models [Figs. 17(c) and (d)]. These results confirm that the wire is a Luttinger liquid in the three-leg NLM but a half-filled Hubbard chain in the 3D wire-substrate model in this particular parameter regime.

The differences between the three-leg NLM and the 3D wire-substrate model in Figs. 16 and 17 can also be seen as an illustration of the failure of the NLM approximation for metallic substrates found in [1]. We see here that not only the substrate properties but also the wire properties are not reproduced correctly by the NLM. Note, however, that the discrepancies are essentially due to the strong dependence of the effective substrate band gap $\Delta_{\mathrm{S}}\left(N_{\mathrm{leg}}\right)$, and thus of the critical coupling $U_{c}$, on the number of legs in the NLM. So a possible remedy could be to rescale $\Delta_{\mathrm{s}}\left(N_{\text {leg }}\right)$ [i.e, to change the rung hoppings $t_{n}^{\text {rung }}$ for $n \geq 2$ in the Hamiltonian (4)].

We conclude that in the doped three-leg NLM a transition occurs from a correlated quasi-1D gapless phase (Luttinger liquid) for $U \lesssim U_{c}$ to a doped band insulator 


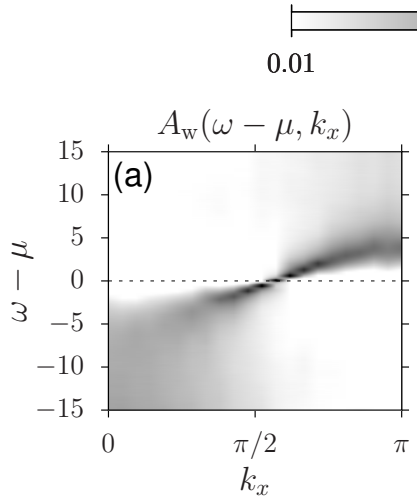

0.1
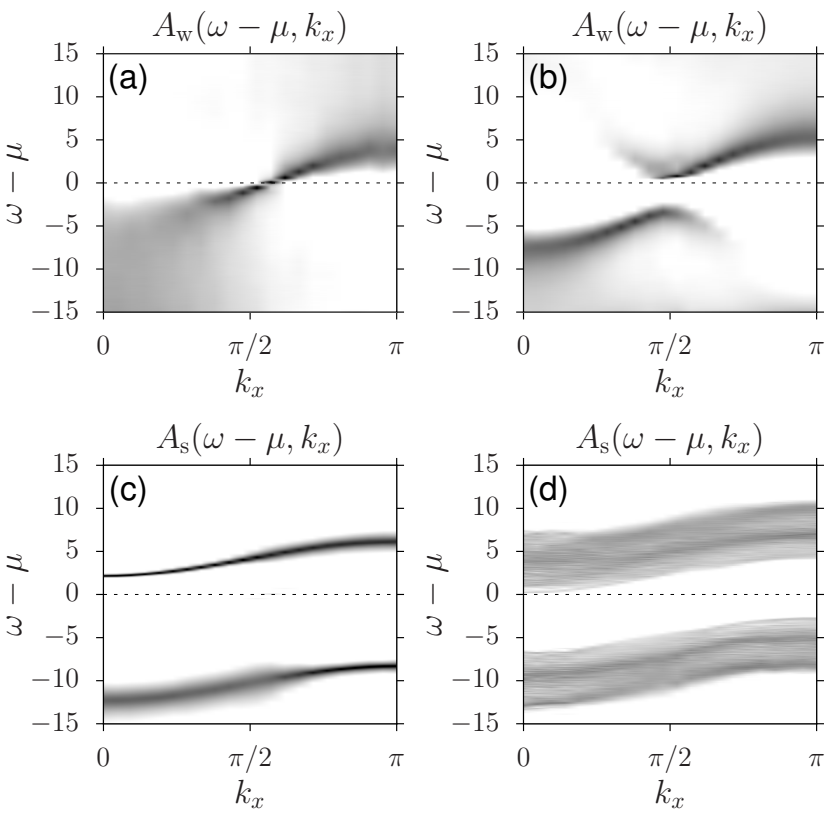

FIG. 16. CT-INT results for the spectral functions $A_{\mathrm{w}}\left(\omega, k_{x}\right)$ $[(\mathrm{a}),(\mathrm{b})]$ and $A_{\mathrm{s}}\left(\omega, k_{x}\right)[(\mathrm{c}),(\mathrm{d})]$ for $U=12, \beta=10$, and $L_{x}=42$. Panels (a) and (c) show results for the three-leg NLM, panels (b) and (d) for the 3D wire-substrate model $\left(L_{y}=42, L_{z}=10\right)$. The chemical potential was $\mu=3.08$ for the NLM and $\mu=1.205$ for the 3D model, corresponding to a doping of $y_{\mathrm{w}} \approx 12.5 \%$.

for $U \gtrsim U_{c}$. The DMRG results for $N_{\text {leg }}>3$ and the QMC spectra suggest that this transition is not an artifact of the NLM but a feature of the 3D wire-substrate model that is qualitatively reproduced by the NLM. This transition between $1 \mathrm{D}$ and $3 \mathrm{D}$ metallic phases is consistent with the transition from a quasi-1D Mott insulator to a $3 \mathrm{D}$ band insulator found at $U_{c}$ for half-filling.

Isolated correlated 1D conductors are Luttinger liquids 9 11. As the NLM with a finite number of legs is a quasi-1D system, it is not surprising that we find a gapless Luttinger liquid phase. However, it is far from obvious that another metallic phase would occur. On the one hand, the existence of the Luttinger liquid phase for $U \lesssim U_{c}$ is fully supported by the confinement of lowenergy excitations to the wire (Figs. 12 and 14) and their clear quasi-1D finite-size scaling (Fig. 10) with distinct charge and spin velocities (Figs. 11 and 15). On the other hand, the existence of the uncorrelated metallic substrate phase for $U \gtrsim U_{c}$ is inferred from the disappearance of these features, in particular the delocalization of excitations on the noninteracting substrate legs and the equality of finite-size charge, spin, and singleparticle gaps. While we could also interpret the metallic substrate phase of few-leg NLMs as a Luttinger liquid with very weak effective interactions, such an interpreta-

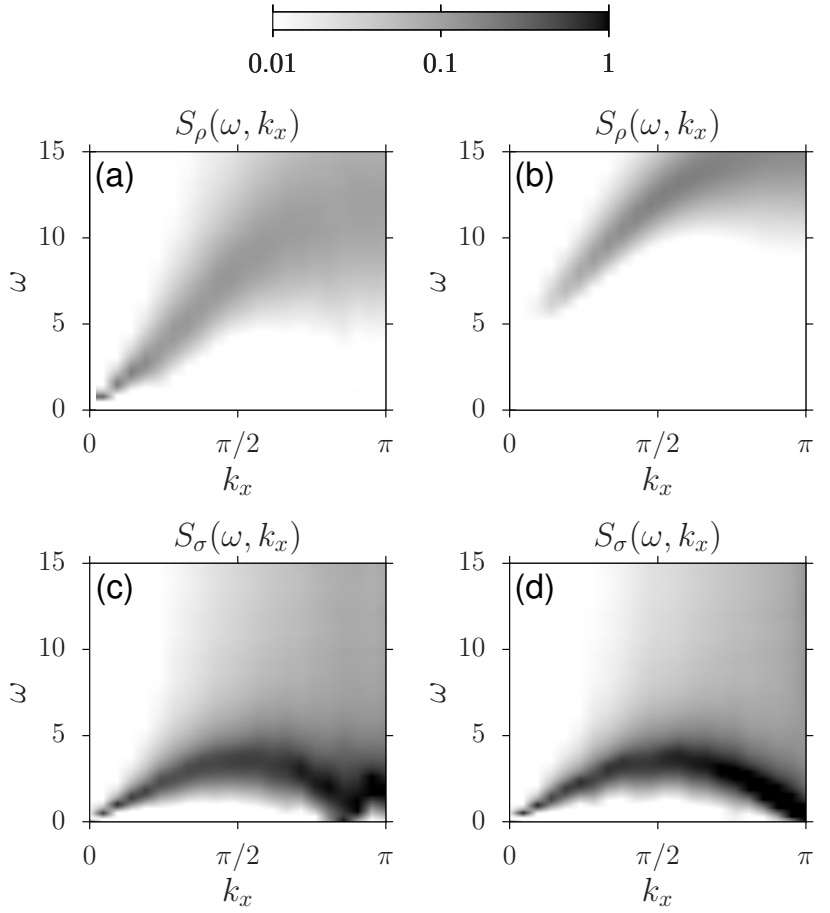

FIG. 17. CT-INT results for the dynamic charge structure factor $S_{\rho}\left(\omega, k_{x}\right)[(\mathrm{a}),(\mathrm{b})]$ and the dynamic spin structure factor $S_{\sigma}\left(\omega, k_{x}\right)[(\mathrm{c}),(\mathrm{d})]$ on the wire for the same parameters as in Fig. 16. Panels (a) and (c) show results for the three-leg NLM, panels (b) and (d) for the 3D wire-substrate model.

tion breaks down in the limit $N_{\text {leg }} \gg 1$ and hence in the 3D wire-substrate model.

Finally, we note that the differences between spin and charge velocities in the Luttinger liquid phase become smaller with increasing $t_{\mathrm{ws}}$ (see Fig. 11) and thus the distinction between a weakly-coupled Luttinger liquid and a quasi-1D Fermi gas becomes moot in the limit $t_{\mathrm{ws}} \rightarrow \infty$. In contrast, any local measurement on the wire in the doped band insulator, such as the local DOS measured by scanning tunneling spectroscopy, could reveal a 1D Mott insulator, as correlation functions (see Fig. 13) suggest that this state subsists as a correlated chain impurity embedded in the substrate for $U \gtrsim U_{c}$.

\section{CONCLUSIONS}

We investigated a correlated wire with a Hubbard interaction deposited on an insulating substrate using the NLM approach developed in 11. Using the DMRG method, we were able to determine the ground-state properties and gaps of NLMs with different numbers of legs. The CT-INT QMC method was used to obtain the spectral properties of both the three-leg NLM and the 3D wire-substrate model. We found that a three-leg NLM already yields a qualitative description of the low-energy physics of the full 3D wire-substrate system. A quanti- 
tative description (e.g., for the charge and spin velocities of the Luttinger liquids) is possible when the low-energy excitations are localized on the wire and DMRG calculations can be carried out for several numbers of legs. It would certainly be useful to obtain additional information from field-theoretical methods.

We found that Mott-insulating and Luttinger liquid phases, which are possibly relevant for atomic wires on semiconducting substrates, can be observed in the $3 \mathrm{D}$ wire-substrate system and are well captured by the NLM. Transitions from 1D low-energy excitations to low-energy excitations delocalized in the substrate can also be observed in the NLM but, by nature, the results depend quantitatively on the number of legs. While the spectral properties calculated with the CT-INT method confirm that these transitions also occur in the $3 \mathrm{D}$ wire-substrate system, we have not yet obtained accurate results for, e.g., the critical values of the interaction $U$ and the hybridization $t_{\mathrm{ws}}$.

It may be surprising at first to find transitions from 1D correlated phases (Mott insulator, Luttinger liquid) to uncorrelated phases (band insulator, metal) upon increasing the interaction $U$ between electrons or decreasing the hybridization $t_{\mathrm{ws}}$ between wire and substrate. However, it should be realized that we consider only the low-energy excitations and that the latter are not always associated with the strongest coupling in a system. This is easily seen in the limits $U \gg t_{\mathrm{ws}}$ or $t_{\mathrm{ws}} \gg U$.

In conclusion, the $3 \mathrm{D}$ wire-substrate model with a Hubbard-type wire and the corresponding effective narrow ladder models provide us with a promising approach to investigate correlation effects in atomic wires on semiconducting substrates. This approach can be easily generalized to extended Hubbard Hamiltonians and electronphonon models. The model properties can be determined using the CT-INT and DMRG methods and additional information could be obtained using other methods for 1D strongly correlated systems.

\section{ACKNOWLEDGMENTS}

This work was supported by the German Research Foundation (DFG) through SFB 1170 ToCoTronics and the Research Unit Metallic nanowires on the atomic scale: Electronic and vibrational coupling in real world systems (FOR1700, grant No. JE 261/1-1). Some of the DMRG calculations were carried out on the cluster system at the Leibniz Universität Hannover. The authors gratefully acknowledge the computing time granted by the John von Neumann Institute for Computing (NIC) and provided on the supercomputer JURECA [57] at the Jülich Supercomputing Centre.
1 First paper of this series: A. Abdelwahab, E. Jeckelmann, and M. Hohenadler, eprint aXiv:1704.07350

2 F. H. L. Eßler, H. Frahm, F. Göhmann, A. Klümper and V. E. Korepin, The One-Dimensional Hubbard Model (Cambridge University Press, Cambridge, 2005).

3 S. R. White, Phys. Rev. Lett. 69, 2863 (1992).

4 S. R. White, Phys. Rev. B 48, 10345 (1993).

${ }^{5}$ U. Schollwöck, Rev. Mod. Phys. 77, 259 (2005).

${ }^{6}$ E. Jeckelmann, in Computational Many Particle Physics (Lecture Notes in Physics 739), edited by H. Fehske, R. Schneider, and A. Weiße (Springer-Verlag, Berlin, Heidelberg, 2008), p. 597.

7 A. N. Rubtsov, V. V. Savkin, and A. I. Lichtenstein, Phys. Rev. B 72, 035122 (2005).

8 E. Gull, A. J. Millis, A. I. Lichtenstein, A. N. Rubtsov, M. Troyer, and P. Werner, Rev. Mod. Phys. 83, 349 (2011).

9 J. Sólyom, Advances in Physics 28, 201 (1979).

10 T. Giamarchi, Quantum Physics in One Dimension (Oxford University Press, Oxford, 2007).

${ }^{11} \mathrm{~K}$. Schönhammer, Luttinger liquids: the basic concepts in D. Baeriswyl and L. Degiorgi (Eds.), Strong Interactions in Low Dimensions (Kluwer Academic Publishers, Dordrecht, 2004).

12 F. Gebhard, The Mott Metal-Insulator Transition (Springer, Berlin, 1997).

13 M. Springborg and Y. Dong, Metallic Chains / Chains of Metals (Elsevier, Amsterdam, 2007).

14 N. Oncel, J. Phys.: Condens. Matter 20, 393001 (2008).

15 P. C. Snijders and H. H. Weitering, Rev. Mod. Phys. 82, 307 (2010).
16 C. Blumenstein, J. Schäfer, S. Mietke, S. Meyer, A. Dollinger, M. Lochner, X. Y. Cui, L. Patthey, R. Matzdorf, and R. Claessen, Nature Physics 7, 776 (2011).

17 C. Blumenstein, J. Schäfer, S. Mietke, S. Meyer, A. Dollinger, M. Lochner, X. Y. Cui, L. Patthey, R. Matzdorf, and R. Claessen, Nature Physics 8, 174 (2012).

18 K. Nakatsuji and F. Komori, Nature Physics 8, 174 (2012).

19 J. Park, K. Nakatsuji, T.-H. Kim, S. K. Song, F. Komori, and H. W. Yeom, Phys. Rev. B 90, 165410 (2014).

20 N. de Jong, R. Heimbuch, S. Eliens, S. Smit, E. Frantzeskakis, J.-S. Caux, H. J. W. Zandvliet, M. S. Golden, Phys. Rev. B 93, 235444 (2016).

21 Y. Ohtsubo, J. Kishi, K. Hagiwara, P. Le Fèvre, F. Bertran, A. Taleb-Ibrahimi, H. Yamane, S. Ideta, M. Matsunami, K. Tanaka, and S. Kimura, Phys. Rev. Lett. 115, 256404 (2015).

${ }^{22}$ K. Yaji, I. Mochizuki, S. Kim, Y. Takeichi, A. Harasawa, Y. Ohtsubo, P. Le Fèvre, F. Bertran, A. Taleb-Ibrahimi, A. Kakizaki, and F. Komori, Phys. Rev. B 87, 241413 (2013).

${ }^{23}$ K. Yaji, S. Kim, I. Mochizuki, Y. Takeichi, Y. Ohtsubo, P. Le Fèvre, F. Bertran, A. Taleb-Ibrahimi, S. Shin, and F. Komori, J. Phys.: Condens. Matter 28, 284001 (2016).

24 C. Tegenkamp, Z. Kallassy, H. Pfnür, H.-L. Günter, V. Zielasek, and M. Henzler, Phys. Rev. Lett. 95, 176804 (2005).

25 M. Wanke, K. Löser, G. Pruskil, D. V. Vyalikh, S. L. Molodtsov, S. Danzenbächer, C. Laubschat, and M. Dähne, Phys. Rev. B 83, 205417 (2011).

${ }^{26}$ I. K. Dash and A. J. Fisher, J. Phys.: Condens. Matter 13, 5035 (2001). 
27 I. K. Dash and A. J. Fisher, e-print arXiv:condmat/0210611 v1 (2002).

28 A. Abdelwahab, E. Jeckelmann, and M. Hohenadler, Phys. Rev. B 91, 155119 (2015).

29 A.O. Gogolin, A.A. Nersesyan, A.M. Tsvelik, Bosonization and Strongly Correlated Systems, Cambridge University Press 1998.

30 G. Hager, G. Wellein, E. Jeckelmann, and H. Fehske, Phys. Rev. B 71, 075108 (2005).

31 S. Moukouri, Phys. Rev. B 70, 014403 (2004).

32 S. Moukouri and E. Eidelstein, Phys. Rev. B 82, 165132 (2010).

33 J. Bonča, J. E. Gubernatis, M. Guerrero, E. Jeckelmann, and Steven R. White, Phys. Rev. B 61, 3251 (2000).

34 T. Xiang, Phys. Rev. B 53, R10445(R) (1996).

35 S. Nishimoto, E. Jeckelmann, F. Gebhard, and R. M. Noack, Phys. Rev. B 65, 165114 (2002).

${ }^{36}$ G. Ehlers, J. Sólyom, Ö. Legeza, and R. M. Noack, Phys. Rev. B 92, 235116 (2015).

37 J. Motruk, M. P. Zaletel, R. S. K. Mong, and F. Pollmann, Phys. Rev. B 93, 155139 (2016).

${ }^{38}$ G. Ehlers, S. R. White, and R. M. Noack, Phys. Rev. B 95, 125125 (2017).

39 A.M. Tsvelik, Quantum Field Theory in Condensed Matter Physics, Cambridge University Press 2003.

40 C. Zhang, E. Jeckelmann, and S. R. White, Phys. Rev. Lett. 80, 2661 (1998).

41 R. J. Bursill, Phys. Rev. B 60, 1643 (1999).

42 E. Jeckelmann and H. Fehske, Riv. Nuovo Cimento 30, 259 (2007).
43 M. Hohenadler, T. C. Lang, and F. F. Assaad, Phys. Rev. Lett. 106, 100403 (2010).

${ }^{44}$ H. Benthien, F. Gebhard, and E. Jeckelmann, Phys. Rev. Lett. 92, 256401 (2004).

45 H. Benthien and E. Jeckelmann, Phys. Rev. B 75, 205128 (2007).

46 E. Jeckelmann, Progress of Theoretical Physics Supplement 176, 143 (2008).

47 A. Nocera and G. Alvarez, Phys. Rev. E 94, 053308 (2016).

48 A. Nocera, N. D. Patel, J. Fernandez-Baca, E. Dagotto, and G. Alvarez, Phys. Rev. B 94, 205145 (2016).

49 K. S. D. Beach, arXiv:cond-mat/0403055 (2004).

50 M. Aichhorn, H. G. Evertz, W. von der Linden, and M. Potthoff, Phys. Rev. B 70, 235107 (2004).

51 E. Jeckelmann and H. Benthien, in Computational Many Particle Physics (Lecture Notes in Physics 739), edited by H. Fehske, R. Schneider, and A. Weiße (Springer-Verlag, Berlin, Heidelberg, 2008), p. 621.

52 M. Raczkowski, F. F. Assaad, and L. Pollet, Phys. Rev. B 91, 045137 (2015).

53 R. G. Pereira, K. Penc, S. R. White, P. D. Sacramento, and J. M. P. Carmelo, Phys. Rev. B 85, 165132 (2012).

54 A. Abendschein and F. F. Assaad, Phys. Rev. B 73, 165119 (2006).

55 V. Meden and K. Schönhammer, Phys. Rev. B 46, 15753 (1992).

56 L. Markhof and V. Meden, Phys. Rev. B 93, 085108 (2016).

57 Jülich Supercomputing Centre (2016), JURECA: Generalpurpose supercomputer at Jülich Supercomputing Centre, Journal of large-scale research facilities, 2, A62. http://dx.doi.org/10.17815/jlsrf-2-121 